\title{
Die gesetzlichen Ausgleichsleistungen gem. § 45a PBefG im Licht des EuGH-Urteils „Altmark Trans“ ${ }^{\text {( }}$
}

Ausgleichszahlung; Beihilfe; EuGH-Urteil „Altmark Trans“; mittlere Reiseweite; Personenbeförderungsausgleichsverordnung; Personenbeförderungsgesetz; rechtssichere (Teilbereichs-)Ausnahme; Selektivität (Spezifität); Zeitfahrausweise des Ausbildungsverkehrs

Die seit langem bekannten gesetzlichen Ausgleichsleistungen nach § 45a PBefG an Unternehmer für die rabattierte Beförderung Auszubildender im Linienverkehr mit Kraftfahrzeugen nach den $\S \S 42$ und 43 Nr. 2 PBefG erscheinen nach dem EuGH-Urteil „, Altmark Trans " in einem neuen Lichte. Die Entscheidung der Luxemburger Richter hat erhebliche Bedeutung für die (beihilfen-) rechtliche Bewertung von Ausgleichszahlungen der öffentlichen Hand für solche Verpflichtungen, die im allgemeinen Interesse stehen (gemeinwohlorientierte Dienste, ,,gemeinwirtschaftliche Verpflichtungen“). Die Prüfung der besagten Ausgleichszahlungen nach \$ 45a PBefG auf deren Europarechtskonformität erlangt dadurch eine zusätzliche Brisanz, dass sich diese Zahlungen mittlerweile in weiten Bereichen des ÖPNV nicht nur als „,Zuschuss“, sondern geradezu als eigenständige Finanzierungsform darstellen. Sollten sich diese Zahlungen als (gemeinschafts-) rechtswidrig i.S.d. Art. 87 Abs. 1 EG (grundsätzlich unzulässige staatliche Beihilfe) erweisen, wären die Folgen für die betroffenen Unternehmen dramatisch. Zukünftig müssten dann die jeweiligen Zahlungen zunächst bei der Europäischen Kommission notifiziert werden; bis zu deren Genehmigung unterliegen sie dem Durchführungsverbot (vgl. Art. 88 Abs. 3 EG). Darüber hinaus droht eine (vom Konkurrenten einklagbare) Rückzahlungsverpflichtung.

1 Vgl. EuGH (Plenum), Urt. v. 24. Juli 2003, Rs. C280/00 „Altmark Trans“ = NJW 2003, S. $2515=$ NVwZ 2003, S. 1101 = NZBau 2003, S. 503. 


\section{Das „Altmark Trans“-Urteil}

Der Gerichtshof der Europäischen Gemeinschaften (EuGH) hatte im Wege der Vorabentscheidung nach Art. 234 EG über Fragen betreffend die Genehmigung von Verkehrsleistungen $\mathrm{zu}$ entscheiden, die ihm das Bundesverwaltungsgericht (BVerwG) mit Vorlagebeschluss vom 06. April $2000^{2}$ vorlegte.

Die in drei Unterfragen gegliederte Vorlage des BVerwG erfolgte im Revisionsverfahren eines Konkurrentenstreits um die Erteilung von mehreren Genehmigungen im Linienverkehr mit Omnibussen im sachsen-anhaltinischen Landkreis Stendal. Ein Verkehrsunternehmen hatte gegen deren Erteilung an einen Konkurrenten mit dem Argument geklagt, es sei das wirtschaftlichere Unternehmen und benötige nur geringere Zuschüsse. Unstreitig war dabei, dass für den Betrieb der betreffenden Verkehre diverse öffentliche $\mathrm{Zu}-$ schüsse erforderlich waren.

Im Verlaufe des Verfahrens wurde die Rechtssache - angesichts der großen, über den Bereich des öffentlichen Personennahverkehrs hinausgehenden, Bedeutung $-\mathrm{zu}$ einem Grundsatzverfahren bezüglich der gemeinschaftsrechtlichen Zulässigkeit von Ausgleichszahlungen für gemeinwirtschaftliche Verpflichtungen.

Im einzelnen trifft der EuGH mit seinem „Altmark Trans“-Urteil vom 24. Juli 2003 wichtige Feststellungen in Bezug auf das Verhältnis von nationalem Recht, primärem und sekundärem Gemeinschaftsrecht zueinander. Insbesondere die Feststellung des Anwendungsvorrangs der Verordnung (EWG) Nr. 1191/69 i.d.F. der VO (EWG) Nr. 1893/91 (im Folgenden nur: VO 1191/69 n.F.), also von sekundärem Gemeinschaftsrecht, bringt die Klärung einer jahrelang umstrittenen Frage zur Geltung von sekundärem EU-Recht. ${ }^{3}$ Mit anderen Worten richtet sich die Zulässigkeit jeder Zuschussgewährung - unabhängig davon, ob ein Verkehr als eigen- oder gemeinwirtschaftlich zu qualifizieren ist - ausschließlich nach der genannten Verordnung; ein Rückgriff auf das Primärrecht, also insbesondere auf das Beihilferegime der Art. $87 \mathrm{ff}$. EG, ist nicht möglich.

Allerdings sieht Art. 1 Abs. 1 Unterabs. 2 der VO 1191/69 n.F. eine Ausnahmebefugnis vor. Danach darf der nationale Gesetzgeber Unternehmen, deren Tätigkeit ausschließlich auf den Betrieb von Stadt-, Vorort- und Regionalverkehrsdiensten beschränkt ist, vom Anwendungsbereich der Verordnung ausnehmen. Unstrittig war dies in der Bundesrepublik für alle genannten Verkehrsdienste bis zum 31. Dezember 1995 der Fall; seit dem 01. Januar 1996 ordnet $\S 8$ IV 3 PBefG jedoch an: „Soweit eine ausreichende Verkehrsbedienung nicht entsprechend Satz 1 [= eigenwirtschaftlich] möglich ist, ist die Verordnung (EWG) Nr. 1191/69 [...] in der jeweils geltenden Fassung maßgeblich.“

Umstritten ist zum einen, ob darin eine teilweise Ausnahme von eigenwirtschaftlichen Verkehren vom Anwendungsbereich der VO 1191/69 liegt und zum anderen, ob ein Mitgliedstaat überhaupt eine Teilausnahme vornehmen darf oder die betreffenden Verkehrsdienste nur in Gänze ausnehmen darf.

2 Vgl. BVerwG, Beschluss vom 06. April 2000, Az. 3 C 7/99 = NVwZ 2001, S. 320.

3 Einige Autoren sehen darin sogar das wichtigste Ergebnis des Urteils, vgl. Berschin (2004), S. 1 m. w. Nachw. Die Gegenansicht bezweifelt den Anwendungsvorrang der Verordnung, vgl. Berschin, aaO. 
In der Vielzahl der einschlägigen Stellungnahmen wird ohne weiteres davon ausgegangen, dass die Regelung des $\S 8$ IV 3 PBefG eine Teilbereichsausnahme darstellt. ${ }^{4}$ Das BVerwG hat dies nicht in dem besagten Vorlagebeschluss thematisiert, was dafür spricht, dass es ebenfalls eine erfolgte Teilbereichsausnahme unterstellt. Der EuGH hat sich im „Altmark Trans“--Urteil nicht explizit zu diesem Problem geäußert. ${ }^{5}$ Es wird im Folgenden daher davon ausgegangen, dass $\S 8$ IV 3 PBefG eine Ausnahme von der Anwendbarkeit der VO 1191/69 n.F. zugunsten eigenwirtschaftlicher Verkehre konstituiert.

Die generelle Rechtmäßigkeit einer solchen Teilbereichsausnahme wurde vom EuGH ausdrücklich bestätigt. ${ }^{6}$ Dies steht allerdings unter dem Vorbehalt, dass die betreffenden Normen des Personenbeförderungsgesetzes den nach dem Grundsatz der Rechtssicherheit gebotenen Erfordernissen der Bestimmtheit und Klarheit genügen. ${ }^{7}$ Erforderlich ist, dass die Rechtslage für den Einzelnen hinreichend bestimmt und klar ist und ihn in die Lage versetzt, von allen seinen Rechten Kenntnis zu erlangen und diese gegebenenfalls vor den nationalen Gerichten geltend zu machen. ${ }^{8}$ Der EuGH hat Zweifel, ob die in Rede stehende Norm des PBefG diesen Anforderungen entspricht ${ }^{9}$ und überlässt die Beantwortung dieser Frage dem BVerwG ${ }^{10}$ das das Verfahren, nachdem die Verfahrensbeteiligten den Rechtsstreit in der Hauptsache für erledigt erklärt hatten, jedoch durch Beschluss vom 11. Dezember $2003^{11}$ gem. $\S 92$ III VwGO eingestellt hat, ohne sich in der Sache nochmals zu äußern.

In Ermangelung einer höchstrichterlichen Feststellung gibt es seither diverse Stellungnahmen in der Literatur. Nach überwiegender Auffassung erfüllt die fragliche Regelung des $\S 8$ IV PBefG die Voraussetzungen des Grundsatzes der Rechtssicherheit und -klarheit. ${ }^{12}$ Als Konsequenz richtet sich die Zulässigkeit von Zuschüssen bei eigenwirtschaftlichen Verkehrsleistungen nicht nach der VO 1191/69 n.F., sondern unmittelbar am Primärrecht, nämlich dem Beihilferechtsregime des EG-Vertrags (Art. 87 ff. EG).

Art. 87 Abs.1 EG gibt einige Tatbestandsmerkmale vor, die nach ständiger Rechtsprechung kumulativ vorliegen müssen. ${ }^{13}$ Demnach muss es sich erstens um eine staatliche

4 Vgl. Wachinger (2004), S. 27 [34 ff.]; Berschin (2000), S. 469 [472]; KCW-GmbH (2004), S. 30 ff., 49 ff., $68 \mathrm{ff}$.

5 Das Gericht unterstellt - gleichsam als Arbeitshypothese - dass die fragliche Regelung des PBefG eine Teilausnahme darstellt und prüft anschließend, ob eine solche Regelung rechtmäßig wäre, vgl. EuGH, Urt. v. 24. Juli 2003, Rs. C280/00, Rn. 51.

6 Vgl. EuGH, Urt. v. 24. Juli 2003, Rs. C280/00, Rn. 57.

7 Vgl. EuGH, Urt. v. 24. Juli 2003, Rs. C280/00, Rn. 58.

8 Ständige Rechtsprechung, vgl. Urt. v. 23. Mai 1985, Rs. 29/84, Slg. 1985, 1661 Rn. 23 „Kommission/Deutschland“; Urt. v. 09. April 1987, Rs. 363/85, Slg. 1987, 1733 Rn. 7 „Kommission/Italien“; Urt. v. 30. Mai 1991, Rs. C59/89, Slg. 1991, I-2607 Rn. 18 „Kommission/Deutschland”; Urt. v. 19. September 1996, Rs. C236/95, Slg. 1996, I-4459 Rn. 13 „Kommission/Griechenland“.

9 Vgl. EuGH, Urt. v. 24. Juli 2003, Rs. C280/00, Rn. 60.

10 Vgl. EuGH, Urt. v. 24. Juli 2003, Rs. C280/00, Rn. 63.

11 BVerwG, Az. 3 C 28/03.

12 Vgl. Ronellenfitsch (2004), S. 7; Bund-Länder-Fachausschuss (BLFA) (2004), 4.1.; Elste/Wiedemann (2004), S. 9 [10 ff.]; Karnop, DVBl. 2004, S. 160 [162 f.]; Sellmann/Wiemann (2004); Oppenländer Rechtsanwälte (hier: Lenz) (2004); OVG Lüneburg, Urt. v. 16. September 2004, Az. 7 LB 3545/01, Entscheidungsgründe II 1. c); a.A. Wachinger (2004), S. 27 [36 ff.]; Kühling/Wachinger (2003), S. 1202 [1204]; Baumeister (2003), S. 550 [551]; KCW-GmbH (2004), dem sich das Hessische Ministerium für Wirtschaft, Verkehr und Landesentwicklung in seinem umstrittenen Erlass vom 12. März 2004 (Az. V 9 - 66 | 28-59-02) anschließt.

13 Vgl. s. a. EuGH, Urt. v. 24. Juli 2003, Rs. C280/00, Rn. 74 m. w. Nachw. 
Maßnahme oder eine Maßnahme unter Inanspruchnahme staatlicher Mittel handeln, die zweitens „bestimmten“ Unternehmen oder Produktionszweigen zugute kommt und drittens eine „Begünstigung“ für diese Unternehmen oder Produktionszweige darstellt. Nach Bejahung dieser Tatbestandsmerkmale stellt sich dann die Frage, ob viertens die Maßnahme wettbewerbsverfälschende Wirkung hat und fünftens den Handel zwischen den Mitgliedstaaten beeinträchtigt. ${ }^{14}$

Das „Altmark Trans“-Urteil hat besondere Bedeutung für die Bewertung des Tatbestandsmerkmals der Begünstigung. Demnach ist der Beihilfe eigen, dass sie dem Begünstigten einen wirtschaftlichen Vorteil verschafft, der nicht auf einer äquivalenten Gegenleistung beruht. ${ }^{15}$ Zwischen EuGH, Europäischem Gericht erster Instanz (EuG) und Kommission war an dieser Stelle die rechtliche Einordnung von staatlichen Ausgleichszahlungen, die im Zusammenhang mit Leistungen der öffentlichen Daseinsvorsorge stehen, äußerst umstritten. Diesbezüglich bestätigt der EuGH nunmehr seinen bereits im bekannten „Ferring“-Urteil ${ }^{16}$ vertretenen „Ausgleichsansatz“ und entwickelt ihn durch vier Zusatzkriterien weiter. Danach stellen öffentliche Mittelzuflüsse bereits tatbestandlich keine Beihilfe i.S.d. Art. 87 Abs. 1 EG dar, soweit sie als Ausgleich für Gegenleistungen anzusehen sind, die von den begünstigten Unternehmen zur Erfüllung gemeinwirtschaftlicher Verpflichtungen erbracht werden. ${ }^{17}$ Dies setzt voraus, dass

1. das begünstigte Unternehmen tatsächlich mit der Erfüllung gemeinwirtschaftlicher Verpflichtungen betraut wurde und diese Verpflichtungen klar definiert worden sind;

2. die Parameter, anhand deren der Ausgleich berechnet wird, zuvor objektiv und transparent aufgestellt wurden;

3. der Ausgleich nicht über das hinausgeht, was erforderlich ist, um die Kosten der Erfüllung der gemeinwirtschaftlichen Verpflichtungen (unter Berücksichtigung der dabei erzielten Einnahmen und eines angemessenen Gewinns aus der Erfüllung dieser Verpflichtungen) ganz oder teilweise zu decken;

4. die Höhe des erforderlichen Ausgleichs auf der Grundlage einer Analyse der Kosten bestimmt wurde, die ein durchschnittliches, gut geführtes Unternehmen, das so angemessen mit Transportmitteln ausgestattet ist, dass es den gestellten gemeinwirtschaftlichen Anforderungen genügen kann, bei der Erfüllung der betreffenden Verpflichtungen hätte, wobei die dabei erzielten Einnahmen und ein angemessener Gewinn aus der Erfüllung dieser Verpflichtungen zu berücksichtigen sind.

In einem solchen Fall erhalte das betreffende Unternehmen in Wirklichkeit keinen finanziellen Vorteil, so dass der staatliche Mittelzufluss nicht bewirke, dass das Unternehmen gegenüber den mit ihm im Wettbewerb stehenden Unternehmen in eine günstigere Wettbewerbsstellung gelange. ${ }^{18}$ Im Ergebnis entfällt somit beim Vorliegen der genannten vier

14 Vgl. Sánchez Rydelski (2003), S. 58.

15 Vgl. Sánchez Rydelski (2003), S. 63 m. w. Nachw.

16 Vgl. EuGH, Urt. v. 22. November 2001, Rs. C53/00 „Ferring“ = EuZW 2002, S. 48 m. Anm. Ruge = NVwZ 2002, S. 193.

17 Vgl. EuGH, Urt. v. 24. Juli 2003, Rs. C280/00, Rn. 87 ff.

18 Vgl. EuGH, Urt. v. 24. Juli 2003, Rs. C280/00, Rn. 87. 
Kriterien das Tatbestandsmerkmal der „Begünstigung“ i.S.d. Art. 87 Abs. 1 EG, so dass bereits tatbestandlich keine Beihilfe vorliegt.

Diese rechtliche Einordnung einer staatlichen Ausgleichszahlung durch den EuGH hat erhebliche praktische Konsequenzen: Der ebenfalls vertretene „Beihilfenansatz“, wonach tatbestandlich eine Beihilfe i.S.d. Art. 87 Abs. 1 EG vorliegt, ${ }^{19}$ die jedoch unter bestimmten Umständen gerechtfertigt ist, begründet die Prüfzuständigkeit der Europäischen Kommission gem. Art. 88 Abs. 1 EG und eine Notifizierungspflicht gem. Art. 88 Abs. 3 Satz 1 EG. Ein rechtswidriger Verstoß gegen die Pflicht, die Kommission vorab zu informieren, hat ein Durchführungsverbot gem. Art. 88 Abs. 3 Satz 3 EG zur Folge. Bei einer Missachtung dieses Verbotes kann die Kommission anordnen, dass das Beihilfeverfahren ausgesetzt oder eine bereits gewährte Beihilfe vorläufig zurückerstattet wird. ${ }^{20}$ Zudem kann sich unter Umständen ein Wettbewerber im Rahmen einer subventionsrechtlichen Konkurrentenklage auf die Rechtswidrigkeit der Beihilfe berufen. Schließlich ist darauf hinzuweisen, dass Verträge, die gegen das Durchführungsverbot verstoßen, gem. $§ 134$ BGB nichtig sind. Durch den „Ausgleichsansatz“ des EuGH werden einerseits diese aufgeführten Risiken vermieden und andererseits verbleibt damit eine wesentliche beihilferechtliche Prüfungskompetenz weiterhin auf der Ebene der Mitgliedstaaten und der nationalen Gerichte.

Es soll im Folgenden die Vereinbarkeit der gesetzlichen Ausgleichsleistungen für die rabattierte Beförderung Auszubildender nach § 45a PBefG mit Gemeinschaftsrecht geprüft werden. Wie bereits festgestellt, richtet sich die gemeinschaftsrechtliche Zulässigkeit von Zuschüssen für eigenwirtschaftliche Verkehrsleistungen nach den Beihilfebestimmungen des EG-Vertrages (Artikel 87 ff.). ${ }^{21}$ Unstreitig liegt das Tatbestandsmerkmal „staatliche Maßnahme“ vor. Umstritten ist hingegen, ob die gesetzlichen Ausgleichsansprüche gem. § 45a PBefG das Tatbestandsmerkmal der Begünstigung ,bestimmter “ Unternehmen oder Produktionszweige i.S.d. Art. 87 Abs. 1 EG erfüllen (sog. Spezifitätskriterium $^{22}$ ). Erst wenn dies bejaht werden müsste, käme es insofern auf das Tatbestandsmerkmal der „Begünstigung“ und somit auf die vom EuGH entwickelten vier Kriterien an.

19 Vgl. v.a. Generalanwalt Léger, Schlussanträge vom 19. März 2002 und 14. Januar 2003.

20 Vgl. hierzu das 3. Kapitel („Verfahren bei rechtswidrigen Beihilfen“) der Verordnung (EG) Nr. 659/1999. Die „Rückforderungsanordnung“ ist allerdings an bestimmte Kriterien gebunden, vgl. Art. 11 Abs. 2 der Verfahrensverordnung.

21 Es handelt sich vorliegend um eigenwirtschaftliche Verkehrsleistungen. Diese sind gem. § 8 IV 2 PBefG solche, deren Aufwand gedeckt wird durch Beförderungserlöse, Erträge aus gesetzlichen Ausgleichs- und Erstattungsregelungen im Tarif- und Fahrplanbereich - etwa für Schülerverkehre nach $§ 45 \mathrm{a}$ PBefG - sowie ,,sonstige Unternehmenserträge im handelsrechtlichen Sinne“.

22 Das Erfordernis der Spezifität wird in der Literatur auch häufig als „Selektivitätskriterium“ bezeichnet. 


\section{Die rechtliche Bewertung der gesetzlichen Ausgleichs- ansprüche gem. § 45a PBefG}

\section{Allgemeines}

$\S$ 45a PBefG gewährt den Verkehrsunternehmen einen Ausgleich für die betriebswirtschaftlich nicht kostendeckende Beförderung von Personen auf Zeitfahrausweisen des Ausbildungsverkehrs. Die Vorschrift beruht auf der Erwägung, dass von den Unternehmern nicht verlangt werden kann, in voller Höhe die Mindereinnahmen hinzunehmen, die daher rühren, dass die öffentliche Hand aus politischen und sozialen Gründen bei Schülern, Studenten und sonstigen Auszubildenden besonders preiswerte Beförderungsentgelte für angemessen hält. ${ }^{23}$ Ausgleichsschuldner ist gem. § 45a III PBefG das Bundesland, in dessen Gebiet der Verkehr betrieben wird.

Dabei ist anzumerken, dass der ÖPNV in der Bundesrepublik auf einen Kostendeckungsgrad (ohne öffentliche Ausgleichs- und Finanzierungsleistungen) von unter 50 Prozent kommt. Im Lauf der letzten Jahre und Jahrzehnte hat sich daher ein komplexes System staatlicher Fördermittel und Ausgleichszahlungen an die Verkehrsunternehmen entwickelt. In der Summe fließen bundesweit jährlich mehrere Milliarden Euro an öffentlichen Zuwendungen in Infrastruktur und Betrieb des ÖPNV. ${ }^{24}$ Es ist anerkannt, dass die Ausgleichs- und Erstattungsansprüche gem. $§ \S 45 \mathrm{a}$ PBefG und 148 SGB IX in der Bundesrepublik mittlerweile mehr sind als reine ,Zuschüsse“; vielmehr kommt ihnen eine Finanzierungsfunktion des ÖPNV zu.

Die Ausgleichszahlungen nach $\S 45$ a PBefG stellen faktisch zu einem großen Teil eine allgemeine Subvention nicht kostendeckender ÖPNV-Leistungen dar. ${ }^{25}$ Bei vielen Regionalverkehrsgesellschaften liegen die Einnahmen je Ausbildungszeitkarte in der Summe von Tarifeinnahme und Ausgleichszahlung bei rund 150 Prozent einer entsprechenden Erwachsenenzeitkarte. $^{26}$

In ländlichen Gebieten mit dominierendem Schülerverkehr haben sich die Ausgleichszahlungen nach $\S 45 \mathrm{a}$ PBefG zu einer allgemeinen Grundfinanzierung des ÖPNV entwi-

23 Vgl. Bidinger (2004), B § 45a Rn. 1; Fromm u.a. (2001), B § 45a Rn. 1; BVerwG, Urt. v. 18. Juli 2002, 3 C 52/01 m. w. Nachw.

24 Vgl. den aktuellen Bericht der Bundesregierung über die Entwicklung der Kostenunterdeckung im ÖPNV aus 2004: „1998 habe die öffentliche Hand insgesamt 14.322 Millionen Euro für die Sicherstellung des ÖPNV aufgewendet.“ Dem entspricht Bölke u.a. (2003), Tabelle 2, S. 13 m. Verw. a. Rönnau, H.J. u.a., Umweltpolitische Handlungsempfehlungen für die Finanzierung des ÖPNV, UFOPLAN-Projekt 29896152 im Auftrag des Umweltbundesamtes, S. 47, wonach 1997 öffentliche Fördergelder zur ÖPNV/SPNV-Finanzierung in Höhe von 14,45 Mrd. EUR geflossen sind (ohne Saarland).

25 Vgl. Hickmann (2004) Teil 2 (Eckpunkte für Änderungen des Gesetzesrahmens), 2.2 (Staatliche Ausgleichszahlungen für verbilligte Tarife im Ausbildungsverkehr ( $\$ 45$ a Personenbeförderungsgesetz), S. 26. Dort heißt es u.a.: „Diese Ausgleichszahlungen haben sich längst zu einer allgemeinen ÖPNV-Subvention an die Verkehrsunternehmen entwickelt.“"

26 Vgl. Hickmann (2004) Teil 2 (Eckpunkte für Änderungen des Gesetzesrahmens), 2.2 (S. 26); Bölke u.a. (2003) S. 15. 
ckelt. ${ }^{27}$ Bundesweit handelt es sich bei den Ausgleichszahlungen nach $\S 45 \mathrm{a}$ PBefG um eine Summe von ca. 1,1 Mrd. Euro (Stand: 2003). ${ }^{28}$

Abgesehen von der daraus resultierenden verkehrspolitischen Fehlsteuerung, ${ }^{29}$ die im Ergebnis darin besteht, dass die unternehmerische Kreativität der Verkehrsunternehmen in volkswirtschaftlich unsinnige Bahnen gelenkt wird und nicht der Kunde im Mittelpunkt des Unternehmensinteresses steht, sondern die Optimierung von Zuschüssen, ${ }^{30}$ stellt sich generell die Frage der Vereinbarkeit der Ausgleichszahlungen nach $\S 45 \mathrm{a}$ PBefG mit höherrangigem Recht. ${ }^{31}$

\section{Die rechtliche Würdigung}

Die Verfassungsmäßigkeit der genannten Regelungen war in der Vergangenheit verschiedentlich Gegenstand der wissenschaftlichen Betrachtung. Dabei wurde die Vereinbarkeit mit Art. 14 Abs. 1 GG bzw. Art. 12 Abs. 1 GG (ggfls. i.V.m. Art. 3 Abs. 1 GG) diskutiert. $^{32}$

27 Vgl. Bölke u.a. (2003), S. 15; Ebenso Overath/Fiedler (2001), S. 101: „ÖPNV ist ohne die Bezuschussung mit derartigen Leistungen [Ausgleichsleistungen gem. § 45a PBefG bzw. § 6a AEG] insbesondere im ländlichen Raum, kaum finanzierbar (...).

28 Bei den Erstattungsleistungen für die unentgeltliche Beförderung Schwerbehinderter nach $\S \S 145 \mathrm{ff}$. SGB IX handelt es sich insgesamt (Bund + Länder) um eine Summe in Höhe von ca. 0,5 Mrd. Euro. Vgl. Hickmann (2004), Teil 2 (Eckpunkte für Änderungen des Gesetzesrahmens), S. 33; Bölke u.a. (2003) S. 13 (Ausgleichszahlungen nach § 45a PBefG u. § 6a AEG: 0,99 Mrd. EUR; Ausgleichszahlungen Schwerbehindertengesetz: 0,46 Mrd. EUR. Bezugsjahr 1997 ohne Saarland); vgl. auch Szabo (2000), S. 78 m. w. Nachw. (Ausgleichszahlungen nach § 45a PBefG u. § 6a AEG: 1,6995 Mrd. DM; Ausgleichszahlungen Schwerbehindertengesetz: 0,8745 Mrd. DM. Bezugsjahr 1993).

29 Durch das komplizierte Berechnungsverfahren kommt es zu einer im Gesetz angelegten systematischen Überkompensation der eigentlichen Tarifvergünstigung: Der Gesamterlös einer Schülermonatskarte plus Ausgleichszahlung liegt etwa 1,5 mal so hoch wie bei einer Erwachsenen-Monatskarte. Dadurch fixieren sich die Verkehrsunternehmen auf den Schülerverkehr und damit auf eine Kundengruppe, die ohnehin auf den ÖPNV angewiesen ist, was zu Lasten der Kundengruppe der erwachsenen Neukunden, also etwa Umsteiger vom Pkw, geht. Außerdem hängt die Höhe der Ausgleichszahlungen von den zurückgelegten Personenkilometern (Pkm) ab, so dass die Verkehrsunternehmen durch Umwegfahrten ihre Zuschüsse erhöhen können.

30 Vgl. Hickmann (2000), S. 54.

31 Die gesetzlichen Erstattungsansprüche gem. $\S \S 145$ ff. SGB IX werden allgemein als beihilferechtlich unproblematisch angesehen. Gem. § 145 SGB IX werden schwerbehinderte Menschen, die infolge ihrer Behinderung in ihrer Bewegungsfähigkeit im Straßenverkehr erheblich beeinträchtigt oder hilflos oder gehörlos sind, von Unternehmern, die öffentlichen Personenverkehr betreiben, unentgeltlich befördert. Dasselbe gilt für Begleitpersonen und mitgeführte Sachen. § 148 SGB IX regelt die Erstattung der dem Unternehmer dadurch entstehenden Fahrgeldausfälle. Erstattungsschuldner sind gem. § 151 SGB IX der Bund und die Länder nach der dort festgelegten Kostenaufteilung.

32 Zuck betrachtet die Regelungen des § 45a PBefG wegen eines Verstoßes gegen Art. 14 Abs. 1 GG als verfassungswidrig, weil er keinen Anspruch auf Kosten gewährt, die aus der Beförderung von Personen mit Zeitfahrausweisen des Berufsverkehrs entstehen. Außerdem ergebe sich die Verfassungswidrigkeit daraus, dass die pauschalen Kostensätze nicht entsprechend den Grundsätzen der Verhältnismäßigkeit und des Rechtsstaatsprinzips ermittelt worden seien und die Differenzierung dieser Sätze nach Stadtgrößen weder mit dem Prinzip der Verhältnismäßigkeit noch mit Art. 14 Abs. 1 GG vereinbar sei. Darüber hinaus sei § 45a PBefG allein deshalb verfassungswidrig, weil seine Ausgestaltung dazu angetan sei, die Wettbewerbsfähigkeit des privaten Omnibusgewerbes unerträglich einzuschränken; vgl. Zuck (1979). Nach Fromm/Wimmer verstößt $\S 45 a$ PBefG weder gegen Art. 12 Abs. 1 GG noch gegen Art. 14 Abs. 1 GG. Lediglich § 3 II und V PBefAusglV begegnen danach verfassungsrechtlichen Bedenken, vgl. Fromm/Wimmer (1980), S. 619 [625]. Die Verfassungsmäßigkeit des $\S 148$ SGB IX (ehemals $\S 62$ SchwbG) wurde von der Rspr. zuletzt vom 5. Senat des Bundesverwaltungsgerichts bestätigt, vgl. BVerwG, Beschl. vom 17. Januar 2003, Az. 5 B 261/02. 
In der jüngeren Vergangenheit steht - bedingt durch die zunehmende Überlagerung der hergebrachten Rechtsordnung durch das europäische Recht ${ }^{33}$ - die Frage nach der Vereinbarkeit mit Gemeinschaftsrecht im Vordergrund der Diskussion.

Diskutiert wird insbesondere, ob die gesetzlichen Ausgleichsansprüche gem. § 45a PBefG das Tatbestandsmerkmal der Begünstigung „bestimmter “ Unternehmen oder Produktionszweige i.S.d. Art. 87 Abs. 1 EG erfüllen. ${ }^{34}$ Für die Bejahung einer (grundsätzlich unzulässigen) Beihilfe ist die sog. Spezifität des Empfängerkreises erforderlich, d.h. Beihilfen müssen selektiv sein und einem ausdrücklich genannten Unternehmen oder einer aufgrund besonderer Merkmale identifizierbaren Gruppe zugute kommen. Solche Merkmale können z.B. Größe, Sektor, Tätigkeit oder Region sein. Als spezifisch (bzw. selektiv) gilt z.B. eine Regelung, die nicht im gesamten Gebiet eines Mitgliedstaats anwendbar ist, z.B. alle regionalen und sektoralen Beihilferegelungen. ${ }^{35}$

In jedem Fall müssen die Wirkungen der betreffenden staatlichen Maßnahme genau untersucht werden. Auf die Bezeichnung oder die Form kommt es nicht an. Um tatsächlich als allgemein zu gelten, müssen die Maßnahmen nach völlig objektiven Kriterien und vollkommen automatisch ohne jeden Ermessensspielraum auf alle Unternehmen, die im Geltungsbereich der jeweiligen staatlichen Stelle ansässig sind, anzuwenden sein. ${ }^{36}$

Nicht in Betracht kommen daher Maßnahmen genereller Natur, also allgemeine konjunkturpolitische Maßnahmen, von denen alle Unternehmen in einem Mitgliedstaat gleichermaßen profitieren, ${ }^{37}$ solange diese Unternehmen objektive und nichtdiskriminierende Bedingungen für die Vergabe erfüllen und soweit sich daraus nicht die regionale oder sektorale Begünstigung bestimmter Unternehmen oder Wirtschaftszweige ergibt.

Nach der Terminologie des EuGHs ist festzustellen, ob eine staatliche Maßnahme im Rahmen einer bestimmten rechtlichen Regelung geeignet ist, bestimmte Unternehmen oder Produktionszweige i.S.d. Art. 87 Abs. 1 EG gegenüber anderen Unternehmen, die sich im Hinblick auf das mit der betreffenden Maßnahme verfolgte Ziel in einer vergleichbaren tatsächlichen und rechtlichen Situation befinden, zu begünstigen. ${ }^{38}$ Allerdings hat der EuGH das Spezifitätskriterium in einer jüngeren Entscheidung extrem weit ausgelegt. ${ }^{39}$

33 Vgl. Berschin (1997), S. 4.

34 Insofern kommt es (zunächst) nicht auf die vier EuGH-Kriterien an, die (erst) im Rahmen des Tatbestandsmerkmals der „Begünstigung“ geprüft werden.

35 Vgl. Sánchez Rydelski (2003), S. 69 m. w. Nachw.

36 Vgl. Bonkamp (2001), S. 101.

37 Dies ist die Konsequenz aus den Art. 98 ff. EG, wonach die allgemeine Wirtschaftspolitik noch weitgehend im Kompetenzbereich der Mitgliedstaaten verblieben ist. Zwar fordert Art. 99 Abs. 1 EG die Koordinierung der Wirtschaftspolitik der Mitgliedstaaten; die Beschlüsse der Gemeinschaft haben aber nur empfehlenden Charakter.

38 Vgl. EuGH, Urt. v. 08. November 2001 - Rs. C143/99 „Adria-Wien Pipeline“, Rn. 41 m. w. Nachw.

39 Vgl. EuGH, Urt. v. 20. November 2003 - Rs. C126/01 „GEMO SA“. 
Die gesetzlichen Ausgleichsleistungen nach $\S 45 \mathrm{a}$ PBefG kommen grundsätzlich unterschiedslos allen Unternehmen im Inland zugute, die im Geltungsbereich der genannten Gesetze Beförderungsleistungen erbringen. Zu beachten ist dabei, dass die zuständigen Behörden bei der Anwendung der gesetzlichen Vorschriften und bei der Auszahlung keinerlei Ermessen hinsichtlich des „Ob“, des „Wann“ und des „Wie“ haben. Im Fall einer Antragstellung darf die Behörde lediglich die gesetzlichen Voraussetzungen prüfen; ein weitergehendes Auswahl- oder Entschließungsermessen hat sie nicht. Auch ein Haushaltsvorbehalt besteht nicht. Jedem Unternehmen, das die Voraussetzungen erfüllt, steht der Ausgleichsanspruch gleichermaßen und unterschiedslos zu. Alle antragstellenden Verkehrsunternehmen werden unter gleichen tatsächlichen Voraussetzungen zwingend gleich behandelt. Somit wird kein Unternehmen gegenüber einem anderen Unternehmen (selektiv) bevorzugt; das Spezifitätskriterium liegt nicht vor, so dass im Ergebnis die gesetzlichen Ausgleichszahlungen gem. § 45a PBefG keine unzulässigen Beihilfen i.S.d. Art. 87 Abs. 1 EG darstellen. ${ }^{40}$

\section{b) Die Rechtsprechung}

Die Rspr. hat sich - soweit ersichtlich - bislang noch nicht explizit mit der (gemeinschaftsrechtlichen) Beihilferechtsrelevanz der gesetzlichen Ausgleichsleistungen nach $\S 45 \mathrm{a}$ PBefG auseinandergesetzt. Daher kommen den Entscheidungen des VG Stade ${ }^{41}$ und v.a. des OVG Lüneburg ${ }^{42}$ vom September 2004 besondere Bedeutung zu. In beiden Fällen handelt es sich um einen Konkurrentenstreit im personenbeförderungsrechtlichen Genehmigungsverfahren.

Das Verwaltungsgericht Stade stellt in Bezug auf das Spezifitätskriterium fest: „Bei diesen Erträgen [nach $\S \S 45 \mathrm{a}$ PBefG, 148 SGB IX] handelt es sich um Regelungen, die dem ÖPNV-Unternehmen Ansprüche einräumen und die schon deshalb nicht geeignet sind,

40 So die h.M., vgl. Burgbacher (1999), S. 1 [3]: „Die Vorschrift [§ 45a PBefG] wird allgemein, wenngleich mit unterschiedlichen Begründungen, als gemeinschaftsrechtskonform angesehen“. Allerdings verkennt er in Fn. 18, dass Berschin in ZUR 1997, S. 4 [8] diese Auffassung gerade nicht teilt. Vgl. auch Fromm (1994), S. 2366 [2368], der zwar nicht explizit das „Selektivitätskriterium“ anspricht - im Kern führt seine Aussage jedoch zur Verneinung desselben: „Wer in Deutschland auf dem Gebiet des öffentlichen Personenverkehrs tätig ist, hat, gleichgültig, ob in öffentlicher oder privater Hand, gleichgültig auch, ob In- oder Ausländer, einen Anspruch auf Ausgleich bzw. Fahrgelderstattung nach diesen Vorschriften [ $\S 45 \mathrm{a}$ PBefG und $\S \S 59$ ff. SchwbG, jetzt $\S \S 145$ ff. SGB IX]; Fromm (1992), S. 256 [258], wonach im Einzelfall ein Ausgleichsanspruch gem. § 45a PBefG enstehen kann, der höher liegt als 50\% der ungedeckten Kosten. Dies sei bundesrechtlich so lange unbedenklich, als der Erstattungsbetrag ein Verlustausgleich bleibt; Fromm u.a. (2001), § 45a Rn. 1; Elste/Wiedemann (2004), S. 9 [24]; VDV (2003), F (Beihilferechtliche Bewertung der gesetzlichen Ausgleichs- und Erstattungsleistungen im Ausbildungsverkehr und für die Freifahrt schwerbehinderter Menschen), S. 17; Bund-Länder-Fachausschuss (BLFA) (2004), 4.4.3.c). Danach spricht „,vieles dafür, dass im Hinblick auf die Ausgleichsleistungen für den Ausbildungsverkehr nach § 45a PBefG keine wesentlichen beihilferechtlichen Risiken bestehen (...)“; Metz (2003b), S. 24 [25]; Heinze (1996), S. 977 [981], der jedoch wohl zu Unrecht von einer Ausnahmeregelung nach Art. 1 Abs. 5 der VO (EWG) Nr. 1191/69 n.F. ausgeht; Meyer (1999), S. 1409 [1410], die auf Art. 9 der VO (EWG) Nr. 1191/69 n.F. abstellt.

41 VG Stade, Urt. v. 16. September 2004, Az. 1 A 463/03 = NZBau 2005, S. 115 ff.

42 OVG Lüneburg, Urt. v. 16. September 2004 , Az. 7 LB 3545/01 = NZBau 2005, S. 53 ff. 
Wettbewerbsverzerrungen herbeizuführen, weil diese jedem Unternehmen in gleicher Weise eingeräumt werden." Damit stellt sich das VG im Ergebnis auf die Seite der h.M. in der Literatur. ${ }^{43}$ Interessant ist, dass das Gericht anschließend noch feststellt, dass die in Rede stehenden Ausgleichszahlungen nach § 45a PBefG materiell gar keine Förderung des ÖPNV seien. Vielmehr verhalte es sich analog zu den gesetzlichen Erstattungsleistungen nach den $\S \S 145$ ff. SGB IX, wonach es sich um eine Erstattung für den den Schwerbehinderten durch das SGB IX gesetzlich eingeräumten Anspruch auf unentgeltliche Beförderung im öffentlichen Personennahverkehr und damit letztlich um eine $\mathrm{Zu}-$ wendung an die Schwerbehinderten selbst handele. Übertragen auf die Beförderung Auszubildender bedeutet dies, dass das Gericht zunächst von einem Anspruch der Auszubildenden auf eine rabattierte Beförderung ausgeht. ${ }^{44}$ Die Ausgleichsleistungen nach $\S 45 \mathrm{a}$ PBefG gleichen nach Ansicht des Gerichts diesen Anspruch (teilweise) dem betreffenden Verkehrsunternehmen gegenüber aus, so dass es sich letztlich um eine Zuwendung an die Auszubildenden selbst handelt.

Das OVG Lüneburg führt in Bezug auf die Ausgleichszahlungen nach § 45a PBefG (und die Erstattung der durch die unentgeltliche Beförderung Schwerbehinderter entstehenden Fahrgeldausfälle nach $\S \S 145$ III, 148 SGB IX) aus: ,(...) stellen sich auch nicht als wettbewerbsverzerrende Beihilfen im Sinne des $\S 92$ Abs. 1 EG-Vertrag ${ }^{45}$ dar, weil diese Erstattungsregelungen jedem Unternehmer diskriminierungsfrei gewährt werden, der solche Beförderungen durchführt und dafür am Markt kostendeckende Erlöse nicht erzielen kann. ${ }^{46}$

c) Eigener Standpunkt

aa) Bezüglich VG Stade

Soweit das Verwaltungsgericht zum Ausdruck bringen will, dass die gesetzlichen Ausgleichsleistungen gem. § 45a PBefG keine unzulässigen Beihilfen i.S.d. Art. 87 Abs. 1 EG seien, weil Auszubildende selbst nicht als Begünstigungsempfänger einer Beihilfe in

43 Vgl. oben Fn. 40.

44 Ein derartiger Anspruch ergibt sich tatsächlich aus der Tarifvorschrift des $\S 39$ PBefG. Zwar steht $\S 39 \mathrm{PBefG}$ unter dem Prinzip der Eigenwirtschaftlichkeit, aber $\S 39$ II PBefG hat nicht nur die wirtschaftlichen Interessen des Unternehmers, sondern auch das öffentliche Verkehrsinteresse und das Gemeinwohl im Blick und fordert einen Ausgleich dieser zum Teil gegenläufigen Interessen. Daher wird davon ausgegangen, dass eine entsprechende Beförderungsentgeltstaffelung in ihrer Gesamtheit sozial- und verkehrspolitische Gesichtspunkte als im öffentlichen Verkehrsinteresse und im Gemeinwohl liegend berücksichtigt und der Ausgleich über eine Mischkalkulation erfolgt. So hat auch das BVerwG in Bezug auf die Gestaltung des Schülertarifs ausgesprochen, dass die Rücksichtnahme auf das Gemeinwohl dem Verkehrsunternehmer in gewissem sachgemäßen Umfang gebietet, sein Tarifschema innerhalb der Grenzen der wirtschaftlichen Lage des Unternehmens nach Regel-, Sozialund Ermäßigungstarifen zu staffeln; vgl. Bidinger (2004), B § 45a Rn. 1 m. w. Nachw.

45 Jetzt: Art. 87 EG. Der Vertrag zur Gründung der Europäischen Gemeinschaft ist in seiner ursprünglichen Fassung am 01. Januar 1958 in Kraft getreten und durch den am 01. Mai 1999 in Kraft getretenen Amsterdamer Vertrag geändert worden. In dem Zusammenhang fand eine Umnummerierung statt (vgl. Art. 12 des Amsterdamer Vertrages in Verbindung mit den Übereinstimmungstabellen dieses Vertrages).

Vgl. OVG Lüneburg, Urt. v. 16. September 2004 , Az. 7 LB 3545/01, Entscheidungsgründe II 1. b). 
Betracht kämen, bedarf dies einer gründlicheren Analyse. Richtig ist, dass als mögliche Empfänger einer Beihilfe in Art. 87 Abs. 1 EG nur ,,bestimmte Unternehmen oder Produktionszweige“ genannt werden; insofern werden Zuwendungen an private Haushalte grundsätzlich nicht vom Beihilfeverbot umfasst. ${ }^{47}$ Erforderlich ist jedoch in jedem Fall eine Abgrenzung zu der sog. Sozialbeihilfe i.S.d. Art. 87 Abs. 2 lit. a EG, die - im Gegensatz zu allgemeinen Sozialmaßnahmen und Hilfen für einzelne Endverbraucher - nur unter bestimmten Voraussetzungen rechtmäßig ist.

Ob eine „Sozialbeihilfe“ i.S.d. Art. 87 Abs. 2 lit. a EG vorliegt, richtet sich nach dem Kreis der Begünstigten, wobei nur zwei Arten von Sozialbeihilfen denkbar sind: ${ }^{48}$

$>$ unmittelbare Beihilfen an eine bestimmte Gruppe von Endverbrauchern, die sich mittelbar bei bestimmten Unternehmen oder Produktionszweigen auswirken, oder

$>$ unmittelbare Zahlungen an bestimmte Unternehmen oder Produktionszweige, die sich mittelbar bei einer bestimmten Endverbrauchergruppe auswirken, diese also begünstigen. Tritt die begünstigende Wirkung dagegen bei den Unternehmen oder einzelnen Produktionszweigen ein, liegt keine Sozialbeihilfe mehr vor.

Zunächst ist festzuhalten, dass die ,§ 45a-Mittel“ faktisch vom Staat ${ }^{49}$ unmittelbar an das beantragende Verkehrsunternehmen und nicht an die Auszubildenden fließen. Entscheidend ist, bei wem die begünstigende Wirkung eintritt. Begünstigen die Zahlungen die Gruppe der Auszubildenden, liegt - sofern diese Beihilfe „ohne Diskriminierung nach der Herkunft" gewährt wird - eine zulässige Sozialbeihilfe i.S.d. Art. 87 Abs. 2 lit. a EG vor. Tritt die begünstigende Wirkung dagegen bei den Verkehrsunternehmen ein, liegt keine „Sozialbeihilfe“ mehr vor, sondern eine (grundsätzlich unzulässige) Beihilfe i.S.d. Art. 87 Abs. 1 EG. ${ }^{50}$

Vordergründig betrachtet, scheinen die gesetzlichen Ausgleichszahlungen gem. $\S 45 \mathrm{a}$ PBefG die Auszubildenden zu begünstigen, weil diese dadurch in den „Genuss“ der verbilligten Beförderung gelangen. Jedoch ergibt sich, wie bereits festgestellt, ${ }^{51}$ der Anspruch des Auszubildenden auf eine rabattierte Beförderung im Ergebnis aus der Tarifvorschrift des $\S 39$ PBefG und ist daher unabhängig von der gleichzeitigen Gewährung eines (teilweisen) staatlichen Ausgleichs für denjenigen Verlust, den der Unternehmer dadurch erleidet, dass er nicht den vollen Preis eines „Jedermann-Zeitfahrausweises“ erhält. Die begünstigende Wirkung tritt folglich beim Empfänger der Zahlungen ein, nämlich den Verkehrsunternehmen. Es handelt sich daher nicht um eine „Sozialbeihilfe“ gem. Art. 87 Abs. 2 lit. a EG.

47 Vgl. Bonkamp (2001), S. 101 m. w. Nachw.

48 Vgl. Otto (2003), S. 66.

49 D.h. dem betreffenden Bundesland.

50 Vgl. Otto (2003), S. 66, der die Frage aufwirft, ob Art. 87 Abs. 2 lit. a EG aufgrund des Art. 1 Abs. 6 der VO (EWG) 1191/69 n.F. im Bereich des ÖPNV möglicherweise keine eigenständige Bedeutung mehr habe, weil Beihilfen für Tarifermäßigungen zu Gunsten bestimmter sozialer Gruppen bereits durch die VO geregelt würden. Dabei verkennt er jedoch das bereits erwähnte deutsche Modell der Teilbereichsausnahme zugunsten eigenwirtschaftlicher Verkehre, vgl. oben „I. Das „Altmark Trans-Urteil““. Die in Rede stehenden Ausgleichszahlungen gem. § 45a PBefG müssen sich nicht an der VO 1191/69 n.F. messen lassen, sondern an Art. 87 Abs. 1 EG.

51 Vgl. oben Fn. 44. 
Soweit die Aussage des VG Stade, es handele sich materiell um gar keine Förderung des ÖPNV, sondern letztlich um eine Zuwendung an die Auszubildenden selbst, so zu verstehen ist, dass das Gericht von einer Legalausnahme nach Art. 87 Abs. 2 EG (sog. Sozialbeihilfe) ausgeht, kann dem somit nicht gefolgt werden. Vielmehr richtet sich die beihilferechtliche Bewertung nach Art. 87 Abs. 1 EG.

\section{bb) Bezüglich des Tatbestandsmerkmals der „Spezifität“}

Überzeugend ist die offenkundige Verneinung des „Spezifitätskriteriums“ i.S.d. Art. 87 Abs. 1 EG durch das Verwaltungs- und Oberverwaltungsgericht. Dem steht auch nicht die erwähnte EuGH-Entscheidung „GEMO SA“ entgegen, ${ }^{52}$ wonach das Selektivitätskriterium extrem weit ausgelegt wird. Gegenstand des Verfahrens war der kostenlose öffentliche Tierkörperbeseitigungsdienst in Frankreich. Obwohl dieser grundsätzlich jedermann zur Verfügung stand (also etwa auch Privatleuten oder dem Staatsforst), hat das Gericht darin eine selektive Maßnahme zugunsten der Viehzüchter und Schlachthöfe gesehen. Dass die von der fraglichen Maßnahme begünstigten Unternehmen verschiedenen Wirtschaftszweigen angehören, genügt nämlich allein noch nicht, um die Selektivität der Maßnahme und damit ihre Eigenschaft als staatliche Beihilfe zu verneinen. ${ }^{53}$ In einer früheren Entscheidung stellte der EuGH fest, dass eine staatliche Initiative weder aufgrund der großen Zahl der begünstigten Unternehmen noch aufgrund der Verschiedenartigkeit und der Bedeutung der Wirtschaftszweige, zu denen diese Unternehmen gehören, als eine allgemeine wirtschaftspolitische Maßnahme angesehen werden könne. ${ }^{54}$

Darüber hinaus muss die in Rede stehende „GEMO SA“-Entscheidung vor folgendem Hintergrund gesehen werden: Hätte der EuGH lediglich geprüft, ob durch die Befreiung von den Kosten der Tierkörperbeseitigung bestimmte Unternehmen gegenüber anderen Unternehmen im Inland besser gestellt werden, hätte das Gericht das Spezifitätskriterium ohne weiteres verneinen müssen, denn offensichtlich kommt der öffentliche Tierkörperbeseitigungsdienst neben den Tierzüchtern und Schlachthöfen allen Privatleuten, dem Staatsforst und auch manchen Unternehmen wie Zoologischen Gärten sowie manchen Behörden gelegentlich zugute. Es ist jedoch offensichtlich, dass die Befreiung der Fleischerzeugungsbetriebe von den Kosten der Tierkörperbeseitigung unmittelbare positive Auswirkungen auf die Produktionskosten und somit auf den innergemeinschaftlichen Handel hat. ${ }^{55}$ Da nach ständiger Rechtsprechung des EuGH die Qualifizierung einer staatlichen Maßnahme als (unzulässige) Beihilfe jedoch das Vorliegen sämtlicher Tatbestandsmerkmale des Art. 87 Abs. 1 EG verlangt, ${ }^{56}$ müsste der in Rede stehende kostenlose Tierkörperbeseitigungsdienst - trotz offenkundiger Handelsbeeinträchtigung zwischen den Mitgliedstaaten - konsequenterweise als gemeinschaftsrechtskonform bewertet

52 Vgl. oben Fn. 39.

53 Vgl. EuGH, Urt. v. 20. November 2003 - Rs. C126/01 „GEMO SA“, Rn. 39 m. w. Nachw.

54 Vgl. EuGH, Urt. v. 08. November 2001 - Rs. C143/99 „Adria-Wien Pipeline“, Rn. 48 m. w. Nachw.

55 Der französische Fleischerzeuger hat geringere Kosten als sein Konkurrent aus einem Mitgliedstaat ohne kostenlosen Tierkörperbeseitigungsdienst, so dass er das Fleisch günstiger anbieten kann.

56 Vgl. s.a. EuGH, Urt. v. 24. Juli 2003 - Rs. C280/00 „Altmark Trans“, Rn. 74 m. w. Nachw. 
werden. Zur Vermeidung dieses offensichtlich zweckwidrigen und dem Sinn und Zweck der gemeinschaftsrechtlichen Wettbewerbsregeln zuwiderlaufenden Ergebnisses, ist die extensive Auslegung des Tatbestandsmerkmals der Spezifität durch den EuGH m.E. gerechtfertigt. Dies gilt jedoch nur bei Fallgestaltungen wie im vom EuGH entschiedenen Fall.

Im Bereich des ÖPNV liegen die Dinge jedoch insofern anders, als ÖPNV-Verkehrsleistungen nur vor Ort produziert werden können. Dieser Umstand wirkt sich auf die Bewertung des Tatbestandsmerkmals der „Beeinträchtigung des Handelsverkehrs“ i.S.d. Art. 87 Abs. 1 EG aus. Der EuGH versteht unter einer Beeinträchtigung des innergemeinschaftlichen Handels i.S.d. Beihilfeaufsichtsrechts jede durch die Gewährung von Beihilfen hervorgerufene staatliche Einwirkung auf diesen Handel. Dabei kommt es insbesondere darauf an, ob durch die Beihilfe die Einfuhr oder Ausfuhr erschwert oder erleichtert wird. Darüber hinaus kommt es darauf an, ob die Beihilfe sich über das Gebiet des die Beihilfe gewährenden Mitgliedstaats hinaus auf die Konkurrenzsituation von Unternehmen in anderen Mitgliedstaaten auswirkt. ${ }^{57}$ Die in Rede stehenden Ausgleichsansprüche gem. § 45a PBefG kommen - wie gezeigt - grundsätzlich unterschiedslos allen Unternehmen zugute, die im Geltungsbereich des Personenbeförderungsgesetzes Beförderungsleistungen erbringen und zwar unabhängig von ihrer Herkunft. Wenn jedoch inländische und ausländische Unternehmen gleichermaßen durch den gesetzlichen Ausgleichsanspruch für die rabattierte Beförderung von Auszubildenden profitieren, wirkt sich dies nicht auf die Konkurrenzsituation zwischen bundesdeutschen Verkehrsunternehmen und Verkehrsunternehmen aus anderen Mitgliedstaaten aus. Eine (unzulässige) Beeinträchtigung des innergemeinschaftlichen Handels besteht in diesem Fall folglich nicht, so dass es keinerlei rechtliches Bedürfnis nach einer extensiven Auslegung des Tatbestandsmerkmals der Spezifität gibt.

Daher bleibt es beim „,normalen“ Anwendungsmaßstab, wonach zu fragen ist, ob die gesetzlichen Ausgleichsleistungen gem. § 45a PBefG geeignet sind, bestimmte Unternehmen oder Produktionszweige i.S.d. Art. 87 Abs. 1 EG gegenüber anderen Unternehmen, die sich im Hinblick auf das mit der betreffenden Maßnahme verfolgte Ziel in einer vergleichbaren tatsächlichen und rechtlichen Situation befinden, zu begünstigen.

Zunächst ist festzuhalten, dass es sich, obwohl nur Verkehrsunternehmen von der Regelung des $\S 45$ a PBefG profitieren, nicht um eine sektorale Beihilfe handelt. Ziel des Gesetzes ist es - wie gezeigt - Schüler, Studenten und sonstige Auszubildende besonders preiswert zu befördern, wobei die durch diese politische und soziale Entscheidung betroffenen Verkehrsunternehmen dafür einen Ausgleich erhalten sollen. Unternehmen aus einem anderen als dem Verkehrssektor befinden sich offensichtlich im Hinblick auf dieses Ziel nicht in einer vergleichbaren tatsächlichen und rechtlichen Situation, weil schlechterdings die (gewerbliche) Personenbeförderung den Unternehmen des Verkehrssektors vorbehalten ist. Anders formuliert können denklogisch nur Verkehrsunternehmen in die Situation gelangen, einen Auszubildenden rabattiert befördern zu müssen. Daher handelt es sich nicht um eine unzulässige sektorale Beihilfe.

57 Vgl. Sánchez Rydelski (2003), S. 74, 75 m. w. Nachw. 
Darüber hinaus wurde bereits festgestellt, dass alle antragstellenden Verkehrsunternehmen unter gleichen tatsächlichen Voraussetzungen zwingend gleich behandelt werden. Somit wird kein Unternehmen gegenüber einem anderen Unternehmen (selektiv) bevorzugt; das Spezifitätskriterium liegt folglich nicht vor.

Eine andere Auffassung verneint die Gemeinschaftsrechtskonformität der gesetzlichen Ausgleichszahlungen gem. § 45a PBefG. ${ }^{58}$ Zum Teil will man diesen aufgrund der Berechnungsregelungen, die teilweise an unternehmensbezogene Merkmale anknüpfen, selektive Wirkungen beimessen. Da eine Begründung bislang nicht publiziert wurde, soll im Folgenden untersucht werden, um welche unternehmensbezogene Merkmale es sich hierbei handelt und inwiefern diese tatsächlich eine selektive Wirkung entfalten können. Zunächst ist festzuhalten, dass nach Lage der Dinge das Vorliegen des Spezifitätskriteriums hinsichtlich der Anspruchsberechtigung offenkundig verneint werden muss. Eine Ungleichbehandlung käme daher nur hinsichtlich der Anspruchshöhe in Betracht. Die entscheidende Frage lautet daher, ob es durch den gesetzlich vorgegebenen Berechnungsmodus zu einer Diskriminierung eines Verkehrsunternehmens gegenüber einem Konkurrenzunternehmen kommt.

Die Berechnung des Ausgleichs für die Beförderung von Personen mit Zeitfahrausweisen erfolgt gem. § 45a II PBefG. Die Ausgleichs-Formel lautet:

$$
A=0,5 \cdot(z \cdot c \cdot t \cdot w \cdot K s p e z-E)
$$

$$
\begin{aligned}
& \mathrm{z}=\text { Anzahl der verkauften Zeitfahrausweise im Ausbildungsverkehr } \\
& \text { c } \quad=\text { Fahrtenhäufigkeit für einen Zeitfahrausweis je Gültigkeitstag } \\
& \mathrm{t}=\text { Anzahl der Gültigkeitstage für einen Zeitfahrausweis } \\
& \mathrm{w}=\text { Mittlere Reiseweite in Km } \\
& \text { Kspez }=\text { Spezifischer }(\text { Soll-)Kostensatz je Personen-Kilometer }(\mathrm{Pkm}) \\
& \mathrm{E}=\text { Erträge im Ausbildungsverkehr }
\end{aligned}
$$

\section{Abb. 1: Ausgleichsformel}

Quelle: $\quad$ Eigene Darstellung

58 Vgl. Overath/Fiedler (2001), S. 101, die im Ergebnis das Tatbestandsmerkmal der Wettbewerbsverfälschung bejahen; indirekt Baumeister (2003), S. 550 [553], der einen Umbau der Förderung des Bunds und der Länder (Schüler- und Schwerbehindertenmittel) fordert, damit für diese Finanzierungen „,beihilferechtskonforme Zustände im Rahmen der Herstellung des europäischen Binnenmarktes einziehen“; mit Bedenken und Zweifeln Barth (2000), S. 136 und 139; Berschin (1997), S. 4 [8], wonach in der Überkompensation der Differenz Schüler-/Jedermannmonatskarte ein Verstoß gegen Art. 10 ff. der VO (EWG) Nr. 1191/69 n.F. liegt; Otto (2003), S. 134, wonach das Merkmal der Selektivität erfüllt ist, aber im Ergebnis die Ausgleichszahlungen aufgrund Art. 1 Abs. 6 der VO (EWG) Nr. 1191/69 n.F. gerechtfertigt ist; Hessisches Ministerium für Wirtschaft, Verkehr und Landesentwicklung, Konsequenzen aus dem EuGH-Urteil vom 24. Juli 2003 - Rs C-280/00 (Altmark Trans) Auswirkungen auf die Genehmigungs- und Vergabepraxis in Hessen (Erlass vom 12. März 2004), wonach es sich unter dem allgemeinen Hinweis auf die weite Fassung der staatlichen Beihilfen i.S.d. EG-Rechts um Beihilfen i.S.d. Art. 87 Abs. 1 EG handelt; wohl auch Burgbacher (1999), S. 1 [4], der darauf abstellt, dass ein Verstoß gegen Gemeinschaftsrecht vorliegt, wenn und soweit im Einzelfall die kommunale Ebene nicht kostendeckende Leistungen erbringen muss, die das Land bestellt hat. 
Betriebsindividuelle Komponenten stellen dabei die Faktoren ,„Z“ (Anzahl der verkauften Zeitfahrausweise im Ausbildungsverkehr) und „E“ (Erträge im Ausbildungsverkehr) dar. Grundsätzlich $^{59}$ pauschaliert angewendet werden die Faktoren: „c“ (gem. § 3 II PBefAusglV = 2,3 Fahrten), ,„t"60 und , $\mathrm{w}^{\mathrm{6}}$ (gem. $§ 3$ IV PBefAusglV $=5 \mathrm{Km}$, wenn überwiegend Orts- und Nachbarortslinienverkehr, bzw. $8 \mathrm{Km}$, wenn überwiegend sonstiger Linienverkehr (Überlandlinienverkehr) betrieben wird.

Der Faktor Kspez ${ }^{61}$ wird von den Landesregierungen oder den von ihnen per Rechtsverordnung ermächtigten Behörden durch Rechtsverordnung nach Durchschnittswerten einzelner repräsentativer Unternehmen, die sparsam wirtschaften und leistungsfähig sind, pauschal vorgegeben, vgl. § 45a II 2 PBefG.

Fraglich ist, ob diese Tatsache dazu geeignet ist, das Spezifitätskriterium zu bejahen. Dies wäre der Fall, wenn es tatsächlich zu einer Bevorzugung eines Unternehmens gegenüber einem Konkurrenzunternehmen kommt, also nicht alle Unternehmen (die sich im Hinblick auf das mit der Maßnahme verfolgte Ziel in einer vergleichbaren tatsächlichen und rechtlichen Situation befinden) gleich behandelt werden. Das mit der Maßnahme verfolgte Ziel besteht darin, den Unternehmen für die Beförderung von Personen auf Zeitfahrausweisen des Ausbildungsverkehrs einen (begrenzten) Ausgleichsanspruch einzuräumen, weil von den Unternehmen nicht verlangt werden kann, in voller Höhe die Mindereinnahmen hinzunehmen, die daher rühren, dass die öffentliche Hand aus politischen und sozialen Gründen bei Schülern, Studenten und sonstigen Auszubildenden besonders preiswerte Beförderungsentgelte für angemessen hält. ${ }^{62}$

Ein konkretes Beispiel verdeutlicht die Problematik:

Ein Schüler in Nordrhein-Westfalen fährt vom Wohnort zur Schule eine Strecke von 8 Km. Eine Schülermonatskarte kostet 35,00 EUR. Es gelten daher folgende Werte:

59 Ausnahme: Nachgewiesene qualifizierte Abweichung von den Durchschnittswerten, vgl. § 3 V PBefAusglV.

60 Für die Ermittlung der Anzahl der berücksichtigungsfähigen Gültigkeitstage ist von der tariflich geregelten betriebsindividuellen Gültigkeitsdauer des Zeitfahrausweises auszugehen; diese wird durch die in $\S 3$ II $3 \mathrm{PBe}-$ fAusglV genannten Höchstwerte begrenzt: Woche $=6$ Tage, Monat = 26 Tage, Jahr = 240 Tage), vgl. Bidinger, Personenbeförderungsrecht, oben Fn. 23, G § 3 PBefAusglV Rn. 23. Allerdings wurde durch die am 01. Januar 2003 in Kraft getretene Verordnung zur Änderung der Verordnung über den Ausgleich gemeinwirtschaftlicher Leistungen im Straßenpersonenverkehr (PBefAusglV) und zur Änderung der Verordnung über den Ausgleich gemeinwirtschaftlicher Leistungen im Eisenbahnverkehr (AEAusglV) v. 25. November 2002 (BGBl. I S. 4450) $\S 3$ II 3 PBefAusglV mit einem 2. Halbsatz ergänzt. Danach können die pauschalierten Höchstwerte von 6, 26 bzw. 240 Tagen unterschritten werden, „soweit Fahrplanangebote nicht vorhanden sind oder tarifliche Einschränkungen bestehen oder nur ausbildungsnotwendige Tage berücksichtigt werden sollen. " Laut Antwort der Bundesregierung auf eine Kleine Anfrage (Drucksache 15/735 vom 31. März 2003) haben bis dato lediglich die Länder Brandenburg und Schleswig-Holstein ein der dritten Alternative entsprechendes Berechnungsverfahren eingeführt.

61 Spezifischer (Soll-)Kostensatz je Personen-Kilometer (Pkm).

62 Vgl. oben Fn. 31. 


$\begin{aligned} \mathrm{c}= & 2,3 \text { Fahrten (gem. §3 Abs. 2 PBefAusglV) } \\ \mathrm{t}= & 26 \text { Schultage } \\ \mathrm{E}= & 35,00 \text { EUR } \\ \mathrm{w}= & 8 \mathrm{Km} \text { (zur Vereinfachung sind individuelle und durchschnittliche } \\ & \text { Beförderungsweite in diesem Beispielsfall identisch) } \\ \text { Kspez = } & \text { wird vom Land NRW vorgegeben. Gem. VO v. 23. April } 1996^{63} \text { und VO } \\ & \text { v. } 06 . \text { November } 2001^{64} \text { z.B. für Unternehmen, die überwiegend sonstigen } \\ & \text { Linienverkehr (Überlandlinienverkehr) mit Omnibussen betreiben: } \\ & 0,1163 \text { EUR/Pkm }\end{aligned}$

Abb. 2: Beispielsfall Berechnung Ausgleichsanspruch

Quelle: Eigene Darstellung

Es ergibt sich demnach als Ausgleich:

$$
A=0,5 \cdot(2,3 \cdot 26 \cdot 8 \cdot 0,1163-35)=10,31896 €
$$

Es ist festzuhalten, dass jedes Unternehmen, das den betreffenden Schüler befördert hätte, diese Summe als Ausgleich bekommen hätte. Da der Berechnungsfaktor „Kspez“ von den einzelnen Bundesländern festgelegt wird und daher variiert, ergeben sich folgende Ausgleichsleistungen für o.g. Beispielsfall in den einzelnen Bundesländern, wobei von ansonsten identischen Parametern ausgegangen wird:

\begin{tabular}{|l|l|}
\hline Bayern & $0,109 € / \mathrm{Pkm}=8,5728 €$ \\
\hline Bremen & $0,11 € / \mathrm{Pkm}=8,812 €$ \\
\hline Niedersachsen & $0,1104 € / \mathrm{Pkm}=8,90768 €$ \\
\hline Rheinland-Pfalz & $0,1089 € / \mathrm{Pkm}=8,54888 €$ \\
\hline Sachsen-Anhalt & $0,116 € / \mathrm{Pkm}=10,2472 €$ \\
\hline Mecklenburg-Vorpommern & $0,138 € / \mathrm{Pkm}=15,5096 €$ \\
\hline
\end{tabular}

Abb. 3: Höhe der Ausgleichszahlung in ausgesuchten Bundesländern

Quelle: Eigene Darstellung

Trotz der unterschiedlichen Ausgleichshöhe in den jeweiligen Bundesländern wird kein Unternehmen gegenüber einem anderen Konkurrenzunternehmen ungleich behandelt. Das ergibt sich aus der Tatsache, dass die Festlegung des Faktors Kspez. nach Durchschnittwerten einzelner repräsentativer Unternehmen erfolgt, die sparsam wirtschaften 
und leistungsfähig sind.$^{65}$ Damit ist diesbezüglich eine Ungleichbehandlung mit Unternehmen aus einem anderen Bundesland ausgeschlossen. Die unterschiedliche Höhe der durchschnittlichen verkehrsspezifischen Kosten resultiert aus den jeweiligen regionalen Besonderheiten, etwa in Bezug auf räumlich-verkehrlich bezogene Elemente (Ballungsgebiete, Flächenland, etc.) und unternehmensbezogene (Tarifverträge, etc.). Aus der Tatsache, dass ein Unternehmen in NRW als Ausgleichszahlung für den genannten Beispielsfall 10,31896 EUR erhält und das bayerische Konkurrenzunternehmen „nur“ 8,5728 EUR, lässt sich daher keineswegs der Schluss zu, dass es dadurch begünstigt wird. Vielmehr bedeutet dies lediglich, dass die durchschnittlichen verkehrsspezifischen Kosten in NRW etwas höher als in Bayern sind. Eine beihilferechtsrelevante Bevorzugung des nordrhein-westfälischen Unternehmens gegenüber seinem Konkurrenten in Bayern liegt damit jedenfalls nicht vor.

Fraglich ist allerdings, wie sich die gesetzliche Unterteilung in

$>$ pauschale und individuelle mittlere Reiseweiten und

$>$ Unternehmen, die überwiegend Orts- und Nachbarortslinien und solchen, die überwiegend Überlandlinienverkehr betreiben auswirkt.

aaa) Die Problematik der „mittleren Reiseweite“

Der Berechnungsfaktor „,“" beschreibt, welche Entfernung ein Auszubildender im Liniennetz eines Verkehrsunternehmens durchschnittlich zurücklegt. Dabei werden pauschal $5 \mathrm{Km}$ vorgegeben, wenn überwiegend Orts- und Nachbarortslinienverkehr bzw. 8 $\mathrm{Km}$, wenn überwiegend sonstiger Linienverkehr (Überlandlinienverkehr) betrieben wird. ${ }^{66}$ Damit sollen die bestehenden strukturellen Unterschiede hinsichtlich der Reiseweiten im Orts- und Nachbarortslinienverkehr einerseits sowie dem Überlandlinienverkehr andererseits berücksichtigt werden. ${ }^{67}$ Die mittlere Reiseweite bezieht sich auf den Ausbildungsverkehr aller Linienverkehre des Unternehmens, weil nur das Gesamtunternehmen nach $\S 45 \mathrm{a}$ PBefG anspruchsberechtigt ist. ${ }^{68}$

$\S 3$ V PBefAusglV sieht jedoch eine „Härteklausel“ vor. Danach ist eine Korrektur der pauschalierten Durchschnittswerte möglich, wenn die tatsächlichen Werte jeweils um mehr als 25 Prozent abweichen. In diesem Fall wird die tatsächliche, individuelle Reiseweite zugrunde gelegt. Dieser „Schwellenwert“ von 3,75 km / 6,25 km ${ }^{69}$ bzw. $6 \mathrm{~km} / 10$ $\mathrm{km}^{70}$ spielt in der Praxis eine wichtige Rolle.

65 Vgl. § 45a II 2 PBefG.

66 § 3 IV PBefAusglV.

67 Vgl. Begr. zum Reg-Entw., BR-Drucks. 246/77 = VkBl. 1977, 429.

68 Vgl. Bidinger (2004), G § 3 Rn. 72 m. w. Nachw.

69 Überwiegend Orts- und Nachbarortslinienverkehr: pauschal $5 \mathrm{~km}+/-25 \%=3,75 \mathrm{~km}$ bzw. $6,25 \mathrm{~km}$.

70 Überwiegend sonstiger Linienverkehr (Überlandlinienverkehr): pauschal $8 \mathrm{~km}+/-25 \%=6 \mathrm{~km}$ bzw. $10 \mathrm{~km}$. 
Betrachten wir im Folgenden zwei Beispielsfälle:

Beispielsfall 1: Ein Verkehrsunternehmen (Typ: Orts- und Nachbarortsverkehre) befördert 1000 Schüler. Die individuelle Reiseweite beträgt $7 \mathrm{~km}$. Aufgrund der Abweichung um mehr als 25\%, wird dieser Wert für die Berechnung des Ausgleichs gem. § 45a PBefG zugrunde gelegt. Im Rahmen eines Genehmigungs- bzw. Ausschreibungswettbewerbs bewirbt es sich auf Linien, mit der Folge, dass dadurch 500 Schüler mit einer mittleren Reiseweite von $4 \mathrm{~km}$ in den bestehenden Verkehr neu integriert werden müssen. Dies hat folgende Auswirkungen:

\begin{tabular}{|l|c|l|c|}
\hline \multicolumn{2}{|l|}{ Ist-Zustand } & $\begin{array}{l}\text { neu zu integ- } \\
\text { rierende } \\
\text { Schüler }\end{array}$ & Zustand nachher \\
\hline $\begin{array}{l}\text { Für die Berechnung nach } \S 45 \mathrm{a} \\
\text { PBefG zu berücksichtigende km }\end{array}$ & 7 & & 5 \\
\hline individuelle Reiseweite & 7 & 4 & $6^{71}$ \\
\hline Anzahl beförderte Schüler & 1000 & 500 & 1500 \\
\hline
\end{tabular}

\section{Abb. 4: Beispielsfall 1}

Quelle: $\quad$ Eigene Darstellung

Angenommen, es gelten folgende Parameter:

\begin{tabular}{|l|c|}
\hline $\begin{array}{l}\text { Fahrten gem. \& 3 Abs. 2 } \\
\text { PBefAusglV }\end{array}$ & 2,3 \\
\hline $\begin{array}{l}\text { Anzahl der } \\
\text { Gültigkeitstage }\end{array}$ & 26 \\
\hline $\begin{array}{l}\text { Preis } \\
\text { Schülermonatskarte }\end{array}$ & 30,00 EUR \\
\hline Kspez. & 0,11 EUR \\
\hline
\end{tabular}

Abb. 5: Beispielsfall 1, zugrundeliegende Parameter

Quelle: Eigene Darstellung

Bisher erhält das Verkehrsunternehmen für die rabattierte Beförderung eines Schülers als Ausgleich

$$
A=0,5 \cdot(2,3 \cdot 26 \cdot 7 \cdot 0,11-30)=8,023 €
$$

Für die 1000 Schüler also insgesamt 8023,00 EUR.

71 Neue individuelle Reiseweite: (1000 Schüler x $7 \mathrm{~km})+(500$ Schüler x $4 \mathrm{~km}): 1500=6 \mathrm{~km}$. 
Nachdem die 500 „,neuen“ Schüler integriert wurden, steht dem Unternehmen als Ausgleich für die rabattierte Beförderung eines Schülers zu:

$$
A=0,5 \cdot(2,3 \cdot 26 \cdot 5 \cdot 0,11-30)=1,445 €
$$

Für die 1500 Schüler also insgesamt 2167,5 EUR.

Durch das gewonnene Liniennetz sinkt die mittlere Reiseweite des Verkehrsunternehmens unter den Schwellenwert von $6,25 \mathrm{~km}$, so dass der Pauschalwert von $5 \mathrm{~km}$ für sein Gesamtnetz mit nunmehr 1500 Schülern angesetzt werden muss. Obwohl sich die Zahl der beförderten Schüler um 50\% erhöht hat, erzielt das Unternehmen im Ergebnis nur einen etwa $25 \%$ höheren Ertrag. ${ }^{72}$

Besonders dramatisch stellt sich die Situation bei Verkehrsunternehmen mit Reiseweiten knapp über dem Schwellenwert und hohen Schülerzahlen dar, die sich um kompakte Stadtnetze bewerben.

Beispielsfall $2^{73}$ :

\begin{tabular}{|l|c|l|c|}
\hline \multicolumn{2}{|l|}{ Ist-Zustand } & $\begin{array}{l}\text { neu zu integ- } \\
\text { rierende } \\
\text { Schüler }\end{array}$ & Zustand nachher \\
\hline $\begin{array}{l}\text { Für die Berechnung nach § 45a } \\
\text { PBefG zu berücksichtigende km }\end{array}$ & 6,4 & & 5 \\
\hline individuelle Reiseweite & 6,4 & 4 & $6,18^{74}$ \\
\hline Anzahl beförderte Schüler & 10000 & 1000 & 11000 \\
\hline
\end{tabular}

Abb. 6: Beispielsfall 2

Quelle: $\quad$ Eigene Darstellung

Angenommen, es gelten folgende Parameter:

\begin{tabular}{|l|c|}
\hline $\begin{array}{l}\text { Fahrten gem. } § 3 \text { Abs. 2 } \\
\text { PBefAusglV }\end{array}$ & 2,3 \\
\hline Anzahl der & 26 \\
Gültigkeitstage & \\
\hline $\begin{array}{l}\text { Preis } \\
\text { Schülermonatskarte }\end{array}$ & 30,00 EUR \\
\hline Kspez. & 0,11 EUR \\
\hline
\end{tabular}

\section{Abb. 7: Beispielsfall 2, zugrundeliegende Parameter}

Quelle: $\quad$ Eigene Darstellung

72 Einnahmen vorher: 1000 Schüler x 30,00 EUR + 8.023,00 EUR $\S 45 \mathrm{a}-\mathrm{Mittel}=38.023,00 \mathrm{EUR}$. Einnahmen neu: 1500 Schüler x 30,00 EUR + 2.167,50 EUR § 45a-Mittel = 47.167,50 EUR.

73 In Anlehnung an das Extrembeispiel von Overath/Fiedler (2001), S. 101 [103] Tabelle 4, wobei es in der Spalte „IST 45a“ bei „km mittlere Beförderungsweite“ nicht 6,5 sondern 6,4 heißen muss.

74 Neue individuelle Reiseweite: (10000 Schüler x 6,4 km) $+(1000$ Schüler x 4 km) : $11000=6,18 \mathrm{~km}$. 
Bisher erhält das Verkehrsunternehmen für die rabattierte Beförderung eines Schülers als Ausgleich

$$
A=0,5 \cdot(2,3 \cdot 26 \cdot 6,4 \cdot 0,11-30)=6,0496 €
$$

Für alle 10000 Schüler also insgesamt 60.496,00 EUR.

Nachdem die 1000 ,neuen“ Schüler integriert wurden, steht dem Unternehmen als Ausgleich für die rabattierte Beförderung eines Schülers zu:

$$
A=0,5 \cdot(2,3 \cdot 26 \cdot 5 \cdot 0,11-30)=1,445 €
$$

Für alle 11000 Schüler also insgesamt 15.895,00 EUR.

Durch das gewonnene Liniennetz sinkt die mittlere Reiseweite des Verkehrsunternehmens unter den Schwellenwert von $6,25 \mathrm{~km}$, so dass der Pauschalwert von $5 \mathrm{~km}$ für sein Gesamtnetz mit nunmehr 11000 Schülern angesetzt werden muss. Obwohl sich die Zahl der beförderten Schüler um 10 Prozent erhöht hat, erzielt das Unternehmen im Ergebnis einen geringeren Ertrag. ${ }^{75}$

Auf den ersten Blick scheinen die genannten Beispielsfälle für eine Ungleichbehandlung einzelner Verkehrsunternehmen gegenüber ihren Konkurrenzunternehmen i.S.d. „Spezifitätskriteriums“ zu sprechen. Insbesondere in Fällen, in denen es zu einem „Abrutschen“ der mittleren Reiseweite unter den Schwellenwert kommt, ist der Einnahmeverlust vergleichsweise deutlich.

Dennoch gibt es auch einige Argumente, die gegen eine derartige rechtliche Bewertung sprechen. Zunächst ist darauf hinzuweisen, dass die aufgeführten Beispielsfälle nur in einer sehr speziellen Situation dazu geeignet sind, auf eine vermeintliche Ungleichbehandlung von konkurrierenden Verkehrsunternehmen hinzudeuten. Lediglich in der Situation des Auslaufens einer Konzession und im anschließenden Genehmigungs- bzw. Ausschreibungswettbewerb werden die aufgeführten Besonderheiten der Ausgleichsberechnung praktisch relevant, weil es faktisch nur in diesen Fällen zu einer signifikanten Veränderung der entsprechenden Parameter kommt, etwa durch das Hinzukommen einer hohen Zahl von „neuen“ Schülern, deren durchschnittliche Beförderungsweite deutlich von der bisherigen mittleren Reiseweite des Unternehmens abweicht und es somit zu einem Unter- bzw. Überschreiten der Schwellenwerte kommt. ${ }^{76}$

Darüber hinaus handelt es sich - gerade im zweiten Beispielsfall - um einen Extremfall, weil das Unternehmen nur $150 \mathrm{~m}$ über dem relevanten Schwellenwert von 6,25 km liegt. Bereits geringe Abweichungen eines einzelnen Parameters hätten zu einer deutlich unspektakuläreren Ertragsentwicklung geführt. Wenn etwa die individuelle Reiseweite der

75 Einnahmen vorher: 10000 Schüler x 30,00 EUR + 60.496,00 EUR § 45a-Mittel = 360.496,00 EUR. Einnahmen neu: 11000 Schüler x 30,00 EUR + 15.895,00 EUR § 45a-Mittel = 345.895,00 EUR.

76 Dabei wird nicht verkannt, dass es auch durch eine „normale“ Veränderung der relevanten Parameter, etwa durch Schülerumzüge, während der Laufzeit der Konzession zu einem Unter- bzw. Überschreiten der Schwellenwerte kommen kann. Dieser Fall dürfte jedoch nach Lage der Dinge verhältnismäßig selten sein; vielmehr wird sich nach der Erfahrung des täglichen Lebens eine signifikante Veränderung in eine bestimmte Richtung durch einen natürlichen Ausgleichsprozess nivellieren. 
bisherigen Schülerbeförderungen von $6,4 \mathrm{~km}$ auf $6,5 \mathrm{~km}$ ansteigt (z.B. durch Schülerumzüge oder einen Verkehrsumweg), fällt das Unternehmen nach dem Gewinn der Linien und dem damit verbundenen Zuwachs der Schülerbeförderungszahlen mit einer individuellen Reiseweite von nunmehr 6,27 km nicht unter den maßgeblichen Schwellenwert von $6,25 \mathrm{~km}$. Nach dem Anstieg der Schülerbeförderungszahlen auf 11000 erlöst das Unternehmen in diesem Fall 31.346,33 EUR ${ }^{77}$ mehr als zuvor mit 10000 beförderten Schülern. Dies belegt, dass es sich bei dem aufgeführten Beispielsfall um einen Extremfall handelt, der nach Lage der Dinge nur äußerst selten auftreten dürfte und daher keineswegs als repräsentativ angesehen werden darf.

Selbst, wenn der eben aufgeführte Fall tatsächlich eintreten sollte, hätte dies nicht automatisch eine Benachteiligung für das Unternehmen zur Folge. Angesichts der Klarheit und Bestimmtheit der Regelung des § 45a PBefG i.V.m. der PBefAusglV ist es nämlich mit verhältnismäßig einfachen Mitteln möglich, entsprechende Risiken bereits im voraus zu erkennen und diese - etwa durch die Gründung eines Tochterunternehmens - auszuschließen.

Darüber hinaus darf nicht verkannt werden, dass den Nachteilen, die zwangsläufig mit jedweder Pauschalierung einhergehen, bestimmte auf der Hand liegende Vorteile gegenüberstehen. So steht etwa dem (berechenbaren und vermeidbaren) „Risiko“ einer Benachteilung durch die in Rede stehende Regelung neben den aus Unternehmenssicht zu begrüßenden Vereinfachungen auch die entsprechende „Chance“ gegenüber, davon zu profitieren, nämlich im Fall eines knappen Überschreitens des unteren Schwellenwertes, was zu einer Anwendung des günstigeren Pauschalwertes führt.

Im Übrigen wurden schon früh verfassungsrechtliche Bedenken bzgl. der pauschalierten Durchschnittswerte (und der ,25\%-Hürde“) geäußert, ${ }^{78}$ inzwischen wird die Verfassungsmäßigkeit der betreffenden Regelung jedoch nicht mehr beanstandet. ${ }^{79} \mathrm{Zu}$ beachten ist außerdem, dass der Gesetzgeber die Problematik bereits im Vorfeld erkannt hat. In der Begründung seines Entwurfes einer PBefAusglV führt der BMV aus: „(...) Bei der Festlegung der mittleren Reiseweite wird (...) berücksichtigt, dass hinsichtlich der Reiseweiten zwischen dem Orts- und Nachbarortslinienverkehr einerseits und dem Überlandlinienverkehr andererseits strukturelle Unterschiede bestehen. Die (...) festgelegten Reiseweiten beruhen auf sorgfältigen Analysen der beteiligten obersten Landesverkehrsbehörden unter Mitberücksichtigung der für den allgemeinen Linienverkehr vorliegenden amtlichen Daten des Statistischen Bundesamtes. $§ 3$ der Verordnung trägt aber auch dem Umstand Rechnung, dass es Verkehrsunternehmen gibt, bei denen die in $\S 3$ Abs. 2 bis 4 genannten Durchschnittswerte vom typischen Erscheinungsbild erheblich abweichen. Werden die Durchschnittswerte um mehr als 25 vom Hundert überschritten, werden (...) anstelle der Durchschnittswerte die für das Unternehmen gültigen konkreten Werte zugrundegelegt, (...). ${ }^{\text {‘80 }}$

77 Einnahmen vorher: 10000 Schüler x 30,00 EUR + 60.496,00 EUR § 45a-Mittel = 360.496,00 EUR. Einnahmen neu: 11000 Schüler x 30,00 EUR + 61.842,33 EUR $\S 45 a-M i t t e l=391.842,33$ EUR.

78 Vgl. VG Oldenburg, Urt. v. 24. November 1983, Az. 6 VG 242/81.

79 Vgl. Bidinger (2004), G ( PBefAusglV), Einführung, m. w. Nachw.

80 Vgl. BR-Dr 246/77 = VkBl. 1977, 429. 
Endlich ist ein weiteres Argument ins Feld zu führen. § 45a II 2 PBefG ermächtigt die Landesregierungen (bzw. den von diesen ermächtigten Behörden) die „durchschnittlichen verkehrsspezifischen Kosten“ festzulegen. Mit dieser Ermächtigungsgrundlage wird nach Auffassung der Rechtsprechung ein weites normgeberisches Ermessen eingeräumt, mit dem Vereinfachungen, Typisierungen und Pauschalierungen bei der Festsetzung der Kostensätze nicht nur ermöglicht werden, sondern ausdrücklich bezweckt sind. ${ }^{81}$ Wenn aber in Bezug auf die spezifischen (Soll-)Kostensätze je Pkm „Pauschalierungen“ (und damit einhergehend Vereinfachungen) ausdrücklich bezweckt sind, dann muss dies auch für die Festsetzung der mittleren Reiseweite gelten, denn ein sachlicher Grund, weshalb diese beiden Parameter für die Berechnung der Ausgleichszahlung nach § 45a PBefG ungleich behandelt werden dürften, ist nicht ersichtlich.

Trotz gewisser Bedenken ergibt die Gesamtschau der Dinge, dass in der pauschalierten Festlegung der „mittleren Reiseweite“ keine Ungleichbehandlung von bestimmten Unternehmen gegenüber anderen Unternehmen i.S.d. „Spezifitätskriteriums“ gem. Art. 87 Abs. 1 EG liegt.

bbb) Die Problematik der Unterscheidung nach der Art des betriebenen Verkehrs

Analog zu der eben dargestellten Problematik des Unter- bzw. Überschreitens der Schwellenwerte der mittleren Reiseweite, stellt sich die Frage, wie es sich beihilferechtlich in Bezug auf das „Spezifitätskriterium“ auswirkt, dass das Gesetz nach Unternehmen, die überwiegend Orts- und Nachbarortslinienverkehr und solchen, die überwiegend sonstigen Linienverkehr (Überlandlinienverkehr) betreiben, differenziert. Gem. § 3 IV PBefAusglV werden für die mittlere Reiseweite im erstgenannten Fall pauschal $5 \mathrm{Km}$, im letztgenannten Fall $8 \mathrm{Km}$ zugrunde gelegt. Fraglich ist, ob darin eine Ungleichbehandlung von bestimmten Unternehmen gegenüber anderen Unternehmen (die sich im Hinblick auf das mit der betreffenden Maßnahme verfolgte Ziel in einer vergleichbaren tatsächlichen und rechtlichen Situation befinden) liegt.

Die Begriffe „Ortslinienverkehr“ und „Nachbarortslinienverkehr“ existierten im PBefG nur bis Ende 1995. Sie wurden bis zu diesem Zeitpunkt in $\S 13$ II Nr. 2 lit. c a.F. genannt, wobei der „Nachbarortslinienverkehr“ legal definiert wurde als der Verkehr zwischen Nachbarorten oder Teilen von ihnen, wenn diese wirtschaftlich und verkehrsmäßig so miteinander verbunden sind, dass der Verkehr nach Tarifgestaltung und nach gegenwärtiger oder in naher Zukunft zu erwartender Häufigkeit einem Ortslinienverkehr vergleichbar ist. Diese Definition wurde wörtlich in 33 IV PBefAusglV vom 25. November 2002 übernommen. Unter dem nicht in der Verordnung definierten Begriff des „Ortslinienverkehrs" wird ein genehmigter Verkehrsdienst verstanden, der innerhalb der politischen Grenzen einer Stadt oder Gemeinde betrieben wird. Alle sonstigen genehmigten 
Verkehrsdienste, die nicht Orts- oder Nachbarortslinienverkehr sind, zählen zum „sonstigen Linienverkehr“" bzw. „Überlandlinienverkehr“. ${ }^{82}$

In der Regel betreiben Unternehmer sowohl Orts- und Nachbarortslinienverkehr als auch Überlandlinienverkehr. In diesen Mischbetriebsfällen kommt es darauf an, welcher der beiden Verkehrsdienste überwiegt. Je nach dem, ob es der Orts- und Nachbarortslinienverkehr oder der sonstige Linienverkehr ist, sind für den gesamten Ausbildungsverkehr des Unternehmens entweder 5 oder $8 \mathrm{~km}$ anzusetzen. Messzahl für das Überwiegen bildet die im Kalenderjahr angefallene Betriebsleistung, die sich in Zug- und Wagen-Kilometern ausdrückt. ${ }^{83}$

Verdeutlichen wir dies an einem Beispiel:

Ein Verkehrsunternehmen betreibt in Nordrhein-Westfalen im Orts- und Nachbarortslinienverkehr 3 Linien mit 60000 Wagen-Kilometern im Jahr und im sonstigen Linienverkehr (Überlandlinienverkehr) 3 Linien mit 62000 Wagen-Kilometern im Jahr. Da der sonstige Linienverkehr (Überlandlinienverkehr) überwiegt, sind für den gesamten Ausbildungsverkehr dieses Mischbetriebes als mittlere Reiseweite $8 \mathrm{~km}$ zugrunde zu legen.

Im Rahmen eines Genehmigungs- bzw. Ausschreibungswettbewerbs bewirbt es sich auf eine Linie im Stadtverkehr, die etwa 5000 Wagen-Kilometer im Jahr umfasst. Dadurch kommt es nunmehr zu einem Überwiegen des Orts- und Nachbarortslinienverkehrs, so dass jetzt für das gesamte Unternehmen nur noch $5 \mathrm{~km}$ als mittlere Reiseweite anzusetzen sind. Daraus resultiert - isoliert betrachtet - ein nicht unerheblicher Einnahmeausfall.

Allerdings sind in diesem Zusammenhang die sog. durchschnittlichen verkehrsspezifischen Kosten („Kspez“) zu beachten. Als solche gelten gem. § 45a II 2 PBefG die Kostensätze je Personen-Kilometer, die von den Landesregierungen oder den von ihnen ermächtigten Behörden durch Rechtsverordnung nach Durchschnittswerten einzelner repräsentativer Unternehmen, die sparsam wirtschaften und leistungsfähig sind, pauschal festgelegt werden. Entscheidend ist dabei die ausdrücklich vorgesehene Möglichkeit, das Pauschalierungsverfahren zur Festsetzung der (Soll-)Kostensätze / Pkm durch unternehmens- und räumlich-verkehrlich bezogene Elemente zu individualisieren. Mit anderen Worten dürfen unterschiedliche Kostensätze für verschiedene Verkehrsregionen ${ }^{84}$ festgelegt werden. Dies ist - soweit ersichtlich - in allen Ländern außer in den Stadtstaaten Berlin und Hamburg geschehen. Alle (Soll-)Kostensatz-VO der Länder gehen von betrieblichen und verkehrlichen Besonderheiten aus, so dass in der Konsequenz in allen Ländern ein höherer (Soll-)Kostensatz für Unternehmen, die überwiegend Orts- und Nachbarortslinienverkehr betreiben, festgesetzt ist. So gilt bspw. für Nordrhein-Westfalen in Bezug auf den Linienverkehr mit Omnibussen:

82 Vgl. Bidinger (2004), G $§ 3$ Rn. 73.

83 Vgl. Bidinger (2004), G $§ 3$ Rn. 74.

84 Unter Besonderheiten verkehrlicher Art versteht der Gesetzgeber ein typisierendes Eingehen auf räumlich bezogene strukturelle Verkehrsverhältnisse wie Besiedlungsdichte, Bebauungsart, Verkehrsdichte, Wirtschaftsstruktur, die Einfluss auf Art und Umfang einer Verkehrsbedienung und damit auf Kosten und Erträge haben; vgl. Bidinger (2004), B $§ 45 a$ Rn. 55. 


\begin{tabular}{|l|l|}
\hline $\begin{array}{l}\text { Unternehmen, die überwiegend Orts- und Nachbarorts- } \\
\text { linienverkehr betreiben }\end{array}$ & $\begin{array}{l}\text { ab 2002: } \\
0,2486 \text { EUR/Pkm }\end{array}$ \\
\hline $\begin{array}{l}\text { Unternehmen, die überwiegend Orts- und Nachbarorts- } \\
\text { linienverkehr in Gemeinden mit mehr als 100.000 Einwoh- } \\
\text { nern betreiben oder (...) }\end{array}$ & ab 2002: \\
\hline $\begin{array}{l}\text { Unternehmen, die überwiegend sonstigen Linienverkehr } \\
\text { (Überlandlinienverkehr) betreiben }\end{array}$ & $\begin{array}{l}\text { ab 2002: } \\
0,1163 \mathrm{EUR} / \mathrm{Pkm}\end{array}$ \\
\hline
\end{tabular}

Abb. 8: (Soll-)Kostensatz Nordrhein-Westfalen

Quelle: $\quad$ Eigene Darstellung

Im Umkehrschluss bedeutet dies für den Beispielsfall, dass es bei dem betreffenden Verkehrsunternehmen zwar durch die gewonnene Stadtverkehrslinie nunmehr zu einem Überwiegen des Orts- und Nachbarortslinienverkehrs kommt und daher als mittlere Reiseweite nicht mehr $8 \mathrm{~km}$, sondern nur noch $5 \mathrm{~km}$ anzusetzen sind; gleichzeitig ist nun jedoch ein höherer (Soll-)Kostensatz zugrunde zu legen, so dass im Ergebnis das „Abrutschen" auf 5 km durch den höheren (Soll-)Kostensatz aufgefangen wird. Im Übrigen gilt auch hier die „Härteklausel“ des $\S 3$ V PBefAusglV, wonach der individuelle Wert als mittlere Reiseweite angesetzt werden darf, wenn dieser von dem Durchschnittswert um mehr als 25 Prozent abweicht.

In der in Rede stehende Differenzierung nach der Art des betriebenen Verkehrs liegt folglich keine Ungleichbehandlung von bestimmten Unternehmen gegenüber anderen Unternehmen i.S.d. „Spezifitätskriteriums“ gem. Art. 87 Abs. 1 EG.

\section{Fazit}

Insgesamt lässt sich festhalten, dass es sich bei den in Rede stehenden Ausgleichsleistungen nach $\S 45 \mathrm{a}$ PBefG nicht um sog. Sozialbeihilfen gem. Art. 87 Abs. 2 EG handelt. Als Folge dessen sind die genannten Zahlungen am allgemeinen Beihilferegime des Art. 87 Abs. 1 EG zu messen. Diesbezüglich steht fest, dass im Rahmen der gesetzlichen Ausgleichszahlungen nach § 45a PBefG alle Unternehmen bzgl. der Anspruchsberechtigung und Anspruchshöhe gleich behandelt werden. Es handelt sich daher um Maßnahmen genereller Natur, von der alle Unternehmen gleichermaßen profitieren. Somit liegt das sog. Selektivitätskriterium („Spezifitätskriterium“) des Art. 87 Abs. 1 EG nicht vor; der Tatbestand der unzulässigen staatlichen Beihilfe ist folglich nicht erfüllt. Es kommt daher nicht mehr auf die vom EuGH entwickelten vier Kriterien zum Tatbestandsmerkmal der „Begünstigung“" an.

Die gesetzlichen Ausgleichszahlungen nach § 45a PBefG sind folglich mit dem Gemeinschaftsrecht vereinbar und unterliegen weder der Notifizierungspflicht bei der Europäischen Kommission noch der Prüfzuständigkeit der Kommission. 
Norbert Targan and Gordon Neumann, The statutory compensation payments pursuant to $\S 45$ a PBefG in the light of the European Court of Justice judgement in the "Altmark Trans"case

Compensation grants; European Court of Justice judgment „Altmark Trans “case; Law on Passenger Transport Services ("Personenbeförderungsgesetz", "PBefG"); legally certain (partial) exception; middle-distance travelling; Ordinance Governing Passenger Transport Services Compensation Grants; selectivity (specificity); State aids; trainee travel season ticket

The long-known statutory compensation payments pursuant to $\S 45$ a PBefG granted to enterprises for the discounted transport of trainees in regular intercity traffic with motor vehicles pursuant to $\S \S 42$ and 43, No. 2 PBefG appear in a new light after the European Court of Justice judgment in the "Altmark Trans"case. The judgment of the Luxembourg judge has substantial significance for the (subsidy) legal valuation of State compensation for such obligations which are in the general public interest ("common welfare services", "general economic interest obligations"). The examination of the afore-mentioned compensation grants pursuant to $\$ 45$ a PBefG with regard to their conformity with European law achieves an additional explosive force in that these payments, in farreaching areas of public transport, represent in the meantime not only a "subsidy" but instead virtually constitute an independent form of financing. Should these payments be proved to be illegal (in the Community) within the meaning of Art. 87(1) EC Treaty (basically impermissible State aid), the consequences for the affected enterprise would be dramatic. In the future, the respective payments must then be first notified to the European Commission; until their approval, they are subject to a prohibition of implementation (cf. Art. 88 (3) EC Treaty). In addition, a repayment obligation (enforceable by competitors) is threatened.

\section{Literaturverzeichnis}

Barth, Sibylle (2000), Nahverkehr in kommunaler Verantwortung: Der öffentliche Personennahverkehr nach der Regionalisierung, Bielefeld

Barth, Sibylle u.a. (2001), Recht des öffentlichen Personennahverkehrs, Praxis-Handbuch, Neuwied

Baumeister, Hubertus (2003), Der EuGH und die Finanzierung der kommunalen Daseinsvorsorge - vor allem des öffentlichen Nahverkehrs, in: NZBau, Heft 10, S. 550 ff.

Berschin, Felix (1997), Europäisches Recht der Finanzierung im ÖPNV bringt Wettbewerb um die Subventionen, in: ZUR, Heft 1, S. 4 ff.

Berschin, Felix (2004), Europarecht für Finanzierung und Genehmigung des öffentlichen Nahverkehrs, in: WiVerw (Vierteljahresbeilage zum Gewerbearchiv), Heft 1, S. 1 ff.

Bidinger, Helmut (Begr.) (2004), Personenbeförderungsrecht, Kommentar zum Personenbeförderungsgesetz nebst sonstigen einschlägigen Vorschriften (Loseblatt-Sammlung), 2. Aufl. 1971, Stand: 12/04, Berlin

Bölke, Wilfried u.a. (2003), Konzeption zur Finanzierung eines umweltverträglichen öffentlichen Personennahverkehrs (ÖPNV) (Diskussionspapier des Umweltbundesamtes), Stand: Juni 2003

Bonkamp, Josef (2001), Die Bedeutung des gemeinschaftsrechtlichen Beihilfeverbotes für die Beteiligung der öffentlichen Hand an einer Kapitalgesellschaft, Berlin 
Bund-Länder-Fachausschuss (BLFA) (2004), Bericht „zu den Konsequenzen der „Altmark“-Entscheidung des Europäischen Gerichtshofes für die Finanzierungs- und Ausschreibungspraxis im ÖPNV / SPNV in Deutschland“" vom 18. Februar 2004

Bundesregierung (2004), aktueller Bericht über die Entwicklung der Kostenunterdeckung im ÖPNV aus 2004, http://www.bundestag.de/bic/hib/2004/2004_134/03

Burgbacher, Hans-Gerwin (1999), Vergaberegeln und Nahverkehr, in: TranspR, Heft 1, S. 1 ff.

Elste, Günter und Torsten Wiedemann (2004), Auswirkungen des EuGH-Urteils in der Rechtssache Altmark Trans auf die Konzessionierung und Finanzierung im ÖPNV, in: WiVerw (Vierteljahresbeilage zum Gewerbearchiv), Heft 1, S. 9 ff.

Fromm, Günter (1980), Der Ausgleich gemeinwirtschaftlicher Leistungen in der deutschen Verkehrsgesetzgebung, in: TranpR, Heft 1, S. 5 ff.

Fromm, Günter (1992), Die Bedeutung der Verordnung (EWG) Nr. 1893/91 für den Ausgleich gemeinwirtschaftlicher Leistungen in Deutschland, in: TranspR, Heft 7/8, S. 256 ff.

Fromm, Günter (1994), Der Fortbestand des Querverbunds - ein steuerliches Problem?, in: BB, Heft 34, S. 2366 ff.

Fromm, Günter u.a. (2001), Personenbeförderungsrecht, Kommentar, 3. Aufl., München

Fromm, Günter und Karl Wimmer (1980), Zur Frage der Verfassungsmäßigkeit der Regelung des Ausgleichs gemeinwirtschaftlicher Leistungen im Ausbildungsverkehr, in: DVB1. 1980, S. 619 ff.

Heinze, Christian (1996), Zur Rechtsstellung der Unternehmen in dem seit 1. Januar 1996 geltenden Personenbeförderungsrecht, in: DÖV, 49. Jg., Heft 23, S. 977 ff.

Hickmann, Gerd (2000), Mehr ÖPNV für's gleiche Geld?, in: ÖPNV: Luxus oder Sparmodell? ÖV an der Grenze der Finanzierbarkeit?, hrsg. von Monika Herrmann, S. 43 ff.

Hickmann, Gerd (2004), Den Übergang in den Wettbewerb aktiv gestalten, August 2004

KCW GmbH (2004), Zur Anwendbarkeit der VO (EWG) Nr. 1191/69 in Deutschland (redigierte Fassung vom 24. Februar 2004) und ergänzende Stellungnahme zum Gutachten vom 24. Februar 2004, beides abrufbar unter www.Wirtschaft.Hessen.de unter „Verkehr“ und dann ,ÖPNV“

Kühling, Jürgen und Lorenz Wachinger (2003), Das Altmark Trans-Urteil des EuGH - Weichenstellung für oder Bremse gegen mehr Wettbewerb im deutschen ÖPNV?, in: NVwZ, S. $1202 \mathrm{ff}$.

Metz, Reiner (2003a), Eigenwirtschaftlicher Nahverkehr im europäischen Binnenmarkt, in: Der Nahverkehr, Heft 9 , S. $8 \mathrm{ff}$.

Metz, Reiner (2003b), Europäisches Beihilferecht und ÖPNV-Finanzierung in Deutschland, in: Der Nahverkehr, Heft 10, S. 24 ff.

Meyer, Susanne (1999), Die Ausschreibungspflicht im gemeinwirtschaftlichen Linienverkehr und ihre Auswirkungen auf den Bestand der Genehmigung, in: DVB1., S. $1409 \mathrm{ff}$.

Oppenländer Rechtsanwälte (hier: Lenz) (2004), Rechtsgutachten zur Anwendbarkeit der Verordnung Nr. 1191/69 in Deutschland vom 14. Mai 2004

Otto, Michael (2003), Die öffentliche Finanzierung und die Genehmigung des ÖPNV (ÖSPV) im Binnenmarkt, Baden-Baden

Overath, Achim und Lothar Holger Fiedler (2001), § 45a PBefG / § 6a AEG - EG-rechtskonform oder wettbewerbsverfälschend?, in: Verkehr und Technik, Heft 3, S. $101 \mathrm{ff}$.

Rönnau, Hans Joachim u.a. (2001), Umweltpolitische Handlungsempfehlungen für die Finanzierung des ÖPNV, UFOPLAN-Projekt 29896152 im Auftrag des Umweltbundesamtes

Ronellenfitsch, Michael (2004), Eigen- und gemeinwirtschaftliche Verkehre sicher unterscheiden, in: Der Nahverkehr, 22. Jg., Heft 4, S. 7 ff.

Sánchez Rydelski, Michael (2003), Handbuch EU Beihilferecht, Baden-Baden

Sellmann, Klaus-Albrecht und Rolf Wiemann (2004), Gutachten zur Frage der wirksamen Teilbereichsausnahme vom Anwendungsbereich der Verordnung (EWG) Nr. 1191/69 für eigenwirtschaftliche Verkehrsleistungen nach dem Personenbeförderungsgesetz (PBefG) vom 20. April 2004

Sterzenbach, Rüdiger (2003), Gutachterliche Stellungnahme: Öffentlicher Personenverkehr - $§ 45$ a Personenbeförderungsgesetz (PBefG)

Szabo, Oliver (2000), Marktbehauptung des ÖPNV - Mitarbeiter des Verbands Region Stuttgart, in: ÖPNV: Luxus oder Sparmodell? ÖV an der Grenze der Finanzierbarkeit?, hrsg. von Monika Hermann, S. 71 ff.

VDV (2003), ÖPNV-Finanzierung nach dem EuGH-Urteil vom 24. Juli 2003

Wachinger, Lorenz (2004), Der Marktzugang im straßengebundenen ÖPNV nach dem EuGH-Urteil in der Rechtssache „Altmark Trans“, in: WiVerw (Vierteljahresbeilage zum Gewerbearchiv), Heft 1, S. 27 ff.

Zuck, Holger (1979), Rechtsgutachten zur Frage der Verfassungsmäßigkeit der Abgeltung gemeinwirtschaftlicher Leistungen nach $\S 45 \mathrm{a}$ PBefG, erstattet für den Verband Baden-Württembergischer Omnibusunternehmer $(\mathrm{WBO})=$ Heft 7 der Schriftenreihe des Bundesverbandes des Deutschen Personenverkehrsgewerbes e.V. (BDP) 